\title{
Genomic and functional analysis of Romboutsia ilealis CRIB' reveals adaptation to the small intestine
}

\author{
Jacoline Gerritsen ${ }^{1,2}$ ， Bastian Hornung ${ }^{\text {Corresp., }} 1,3$ ， Bernadette Renckens ${ }^{4}$, Sacha A.F.T. van Hijum ${ }^{4,5}$, Vitor A.P. \\ Martins dos Santos ${ }^{3,6}$, Ger T. Rijkers ${ }^{7,8}$ ， Peter J. Schaap ${ }^{3}$ ， Willem M. de Vos ${ }^{1,9}$ ， Hauke Smidt ${ }^{1}$ \\ ${ }^{1}$ Laboratory of Microbiology, Wageningen University \& Research, Wageningen, The Netherlands \\ 2 Winclove Probiotics, Amsterdam, The Netherlands \\ 3 Laboratory of Systems and Synthetic Biology, Wageningen University \& Research, Wageningen, The Netherlands \\ 4 Nijmegen Centre for Molecular Life Sciences, CMBI, Radboud UMC, Nijmegen, The Netherlands \\ 5 NIZO, Ede, The Netherlands \\ 6 LifeGlimmer GmbH, Berlin, Germany \\ 7 Laboratory for Medical Microbiology and Immunology, St. Antonius Hospital, Nieuwegein, The Netherlands \\ 8 Department of Science, University College Roosevelt, Middelburg, The Netherlands \\ 9 Departments of Microbiology and Immunology and Veterinary Biosciences, University of Helsinki, Helsinki, Finland \\ Corresponding Author: Bastian Hornung \\ Email address: bastian.hornung@gmx.de
}

\section{Abstract}

Background. The microbiota in the small intestine relies on their capacity to rapidly import and ferment available carbohydrates to survive in a complex and highly competitive ecosystem. Understanding how these communities function requires elucidating the role of its key players, the interactions among them and with their environment/host.

Methods. The genome of the gut bacterium Romboutsia ilealis $\mathrm{CRIB}^{\top}$ was sequenced with multiple technologies (Illumina paired-end, mate-pair and PacBio). The transcriptome was sequenced (Illumina HiSeq) after growth on three different carbohydrate sources, and short chain fatty acids were measured via HPLC.

Results. We present the complete genome of Romboutsia ilealis $\mathrm{CRIB}^{\top}$, a natural inhabitant and key player of the small intestine of rats. $R$. ilealis $\mathrm{CRIB}^{\top}$ possesses a circular chromosome of 2,581,778 bp and a plasmid of 6,145 bp, carrying 2,351 and eight predicted protein coding sequences, respectively. Analysis of the genome revealed limited capacity to synthesize amino acids and vitamins, whereas multiple and partially redundant pathways for the utilization of different relatively simple carbohydrates are present.

Transcriptome analysis allowed identification of the key components in the degradation of glucose, L-fucose and fructo-oligosaccharides.

Discussion. This revealed that $R$. ilealis $\mathrm{CRIB}^{\top}$ is adapted to a nutrient-rich environment where carbohydrates, amino acids and vitamins are abundantly available. 
1 Genomic and functional analysis of Romboutsia ilealis $\mathrm{CRIB}^{\mathrm{T}}$ reveals adaptation to the

2 small intestine

3 Jacoline Gerritsen ${ }^{1,2^{*}}$, Bastian Hornung ${ }^{1,3^{*}}$, Bernadet Renckens ${ }^{4}$, Sacha A. F. T. van Hijum ${ }^{4,5}$, Vitor

4 A.P. Martins dos Santos $^{3,6}$, Ger T. Rijkers ${ }^{7,8}$, Peter J. Schaap ${ }^{3}$, Willem M. de Vos ${ }^{1,9}$ and Hauke

5 Smidt $^{1}$

$6{ }^{1}$ Laboratory of Microbiology, Wageningen University \& Research, Stippeneng 4, 6708 WE,

7 Wageningen, the Netherlands;

$8{ }^{2}$ Winclove Probiotics, Hulstweg 11, 1032 LB, Amsterdam, the Netherlands;

$9{ }^{3}$ Laboratory of Systems and Synthetic Biology, Wageningen University \& Research, Stippeneng

10 4, 6708 WE, Wageningen, the Netherlands;

$11{ }^{4}$ Nijmegen Centre for Molecular Life Sciences, CMBI, Radboud UMC, P.O. Box 9101, 6500

12 HB, Nijmegen, the Netherlands;

$13{ }^{5}$ NIZO, Kernhemseweg 2, 6718 ZB, Ede, the Netherlands;

$14{ }^{6}$ LifeGlimmer GmbH, Markelstrasse 38, Berlin, Germany;

$15{ }^{7}$ Laboratory for Medical Microbiology and Immunology, St. Antonius Hospital, P.O. Box 2500,

163430 EM, Nieuwegein, the Netherlands;

$17{ }^{8}$ Department of Science, University College Roosevelt, P.O. Box 94, 4330 AB, Middelburg, the 18 Netherlands;

$19{ }^{9}$ Departments of Microbiology and Immunology and Veterinary Biosciences, University of

20 Helsinki, P.O. Box 66, 00014, University of Helsinki, Finland

$21{ }^{*}$ These authors contributed equally to this work

22 \#Corresponding author:

23 Bastian Hornung ${ }^{1,3}$ 
24 Email address: bastian.hornung@gmx.de 


\section{Abstract}

26 Background. The microbiota in the small intestine relies on their capacity to rapidly import and

27 ferment available carbohydrates to survive in a complex and highly competitive ecosystem.

28 Understanding how these communities function requires elucidating the role of its key players,

29 the interactions among them and with their environment/host.

30 Methods. The genome of the gut bacterium Romboutsia ilealis $\mathrm{CRIB}^{\mathrm{T}}$ was sequenced with 31 multiple technologies (Illumina paired-end, mate-pair and PacBio). The transcriptome was 32 sequenced (Illumina HiSeq) after growth on three different carbohydrate sources, and short chain 33 fatty acids were measured via HPLC.

34 Results. We present the complete genome of Romboutsia ilealis $\mathrm{CRIB}^{\mathrm{T}}$, a natural inhabitant and

35 key player of the small intestine of rats. $R$. ilealis $\mathrm{CRIB}^{\mathrm{T}}$ possesses a circular chromosome of $362,581,778 \mathrm{bp}$ and a plasmid of $6,145 \mathrm{bp}$, carrying 2,351 and eight predicted protein coding 37 sequences, respectively. Analysis of the genome revealed limited capacity to synthesize amino 38 acids and vitamins, whereas multiple and partially redundant pathways for the utilization of 39 different relatively simple carbohydrates are present. Transcriptome analysis allowed 40 identification of the key components in the degradation of glucose, L-fucose and fructo41 oligosaccharides.

42 Discussion. This revealed that $R$. ilealis $\mathrm{CRIB}^{\mathrm{T}}$ is adapted to a nutrient-rich environment where 43 carbohydrates, amino acids and vitamins are abundantly available.

\section{Introduction}

45 Intestinal microbes live in a complex and dynamic ecosystem, and to survive in this highly 46 competitive environment, they have developed close (symbiotic) associations with a diverse array 47 of other intestinal microbes and with their host. This has led to a complex network of host48 microbe and microbe-microbe interactions in which the intestinal microbes and the host co- 
49 metabolise many substrates (Backhed et al. 2005; Scott et al. 2013). In addition to competition

50 for readily available carbohydrates in the diet, intestinal microbes are able to extract energy from

51 dietary polysaccharides that are indigestible by the host (Flint et al. 2012). Furthermore, intestinal

52 microbes can utilize host-derived secretions (e.g. mucus) as substrates for metabolic processes

53 (Ouwerkerk et al. 2013). In turn, the metabolic activities of the intestinal microbes result in the

54 production of a wide array of compounds, of which some are important nutrients for the host. For

55 example, short chain fatty acids (SCFA), the main end-products of bacterial fermentation in the

56 gut, can be readily absorbed by the host and further metabolized as energy sources (Elia \&

57 Cummings 2007) (Lange et al. 2015). All together, the metabolic activity of the intestinal

58 microbiota has a major impact on the health of the host, and recent studies have indicated an

59 important role for microbial activity in diseases such as inflammatory bowel disease, irritable

60 bowel syndrome and obesity (Gerritsen et al. 2011a; Quigley 2013).

61 We only have a limited understanding of the heterogeneity in microbial community composition

62 and activity in different niches along the length of the intestinal tract. To unravel the functional

63 contribution of specific intestinal microbes to host physiology and pathology, we have to

64 understand their metabolic capabilities at a higher resolution. It is still difficult, however, to

65 associate a functionality in this ecosystem to specific sets of genes and in turn to individual

66 microbial species, and vice versa. To this end, the combination of genome mining and functional

67 analyses with single microbes or with simple and defined communities can provide an overall

68 insight in the genetic and functional potential of specific members of the intestinal microbial

69 community (Heinken et al. 2013; Li et al. 2008; Xu et al. 2003).

70 As mentioned above, intestinal microbes have adapted or even specialized in foraging certain

71 niche-specific substrates. However, little is known about the adaption of intestinal microbes to

72 the conditions in the small intestine (Booijink et al. 2007; van den Bogert et al. 2013b; Zhang et

73 al. 2014). Community composition and activity in the small intestine is largely determined by the 
74 host digestive fluids such as gastric acid, bile and pancreatic secretions. The small intestine is a

75 nutrient-rich environment, and previous studies have shown that the microbial communities in the

76 (human) small intestine are driven by the rapid uptake and conversion of simple carbohydrates

77 (Zoetendal et al. 2012; Leimena et al. 2013). Genomic studies of small intestinal isolates have

78 indicated environment-specific adaptations to the small intestine with respect to their

79 carbohydrate utilization capacities, which was evidenced by the presence of a wide array of genes

80 involved in nutrient transport and metabolism of, mainly simple, carbohydrates (Van den Bogert

81 et al. 2013a).

82 Here we describe a model driven genomic analysis of the small intestinal inhabitant Romboutsia

83 ilealis $\mathrm{CRIB}^{\mathrm{T}}$ (Gerritsen et al. 2014). R. ilealis $\mathrm{CRIB}^{\mathrm{T}}$ is currently still the only isolate of the

84 recently descibed species $R$. ilealis, a species that belongs to the family Peptostreptococcaceae,

85 of which many members are common intestinal microbes including the well-known species

86 Clostridioides difficile (previously known as Clostridium difficile) and Intestinibacter bartlettii

87 (previously known as Clostridum bartlettii) (Galperin et al., 2016). An overview of the metabolic

88 capabilities and nutritional potential of the type strain of $R$. ilealis $\mathrm{CRIB}^{\mathrm{T}}$ is provided here to

89 identify potential mechanisms that enable this organism to survive in the competitive small

90 intestinal environment.

\section{Materials and methods}

\section{Genome sequencing, assembly and annotation}

93 R. ilealis $\mathrm{CRIB}^{\mathrm{T}}$ (DSM 25109) was routinely cultured in CRIB medium at $37{ }^{\circ} \mathrm{C}$ as previously

94 described (Gerritsen et al. 2014). Genomic DNA extraction was performed as previously 95 described (Van den Bogert et al. 2013a). Genome sequencing was done using 454 Titanium 96 pyrosequencing technology (Roche 454 GS FLX), as well as Illumina (Genome Analyzer II and 
97 HiSeq2000) and PacBio sequencing (PacBio RS). Mate-pair data was generated by BaseClear

98 (Leiden, the Netherlands). All other data was generated by GATC Biotech (Konstanz, Germany).

99 The genome was assembled in a hybrid approach with multiple assemblers. In short, after

100 estimation of the genome size, assembly of the genome was performed with two different

101 assemblers in parallel using the different sequence datasets. After merging the two assemblies

102 three rounds of scaffolding were performed, once with paired-end data and twice with mate-pair

103 data. Gap-filling was performed after each scaffolding step.

104 Genome annotation was carried out with an in-house pipeline. Prodigal v2.5 was used for

105 prediction of protein coding DNA sequences (CDS) (Hyatt et al. 2010), InterProScan 5RC7 for

106 protein annotation (Hunter et al. 2012), tRNAscan-SE v1.3.1 for prediction of tRNAs (Lowe \&

107 Eddy 1997) and RNAmmer v1.2 for the prediction of rRNAs (Lagesen et al. 2007). Additional

108 protein function predictions were derived via BLAST identifications against the UniRef50 (Suzek

109 et al. 2007) and Swissprot (UniProt-Consortium 2014) databases (download August 2013).

110 Afterwards the annotation was further enhanced by adding EC numbers via PRIAM version

111 March 06, 2013 (Claudel-Renard et al. 2003). Non-coding RNAs were identified using

112 rfam_scan.pl v1.04, on release 11.0 of the RFAM database (Burge et al. 2013). CRISPRs were

113 annotated using CRISPR Recognition Tool v1.1 (Bland et al. 2007).

114 Qualitative metabolic modelling has been performed with Pathway tools v18.0 (Latendresse et

115 al., 2012). A generic default medium consisting out of ammonia/urea, sulfite, hydrogen sulfide

116 and phosphate was assumed, and the qualitative possibility to produce all necessary biomass

117 metabolites was tested with the supply of different carbohydrates, which had been tested before

118 in vitro.

119 See the Supplemental Methods in Text S1 for details on the genomic DNA extraction, genome 120 sequencing, assembly, annotation, and metabolic modelling. 


\section{Whole-genome transcriptome analysis}

122 R. ilealis $\mathrm{CRIB}^{\mathrm{T}}$ was grown in a basal bicarbonate-buffered medium (Stams et al. 1993)

123 supplemented with $16 \mathrm{~g} / \mathrm{L}$ yeast extract (BD, Breda, The Netherlands) and an amino acids

124 solution as used for the growth of $C$. difficile (Karasawa et al. 1995). In addition, the medium was

125 supplemented with either $0.5 \%$ (w/v) D-glucose (Fisher Scientific Inc., Waltham, MA USA), L-

126 fucose (Sigma-Aldrich, St. Louis, MO, USA) or fructo-oligosaccharide (FOS) P06 (DP 2-4;

127 Winclove Probiotics, Amsterdam, The Netherlands). The final $\mathrm{pH}$ of the medium was adjusted to

128 7.0. For each condition, triplicate cultures were set up. For RNA-seq analysis, the cells were

129 harvested in mid-exponential phase $\left(\mathrm{OD}_{600 \mathrm{~nm}}=0.25-0.55, \sim 8-10 \mathrm{~h}\right.$ incubation $)$ (Table $\left.\mathrm{S} 1\right)$.

130 Total RNA was purified using the RNeasy Mini Kit (QIAGEN GmbH, Hilden, Germany).

131 Depletion of rRNA was performed using the Rib-Zero ${ }^{\mathrm{TM}}$ Kit for bacteria (Epicentre

132 Biotechnologies, Madison, WI, USA). The ScriptSeq ${ }^{\mathrm{TM}}$ v2 RNA-seq Library Preparation Kit in

133 combination with ScriptSeq ${ }^{\mathrm{TM}}$ Index PCR primers (Epicentre Biotechnologies) was used for

134 library construction for whole-transcriptome sequencing (RNA-seq). The barcoded cDNA

135 libraries were pooled and sent to GATC Biotech (Konstanz, Germany) where 150 bp sequencing

136 was performed on one single lane using the Illumina HiSeq2500 platform in combination with

137 the TruSeq Rapid SBS (200 cycles) and TruSeq Rapid SR Cluster Kits (Illumina Inc., San Diego,

138 CA, USA). Reads were mapped to the genome with Bowtie2 v2.0.6 (Langmead \& Salzberg

139 2012) using default settings, after quality control (rRNA removal, adapter trimming, and quality

140 trimming) had been performed. Details on the RNA-seq raw data analysis can be found in Table

141 S2 and Supplemental Methods in Text S1.

142 Gene expression abundance estimates and differential expression analysis was performed using

143 Cuffdiff v2.1.1 (Trapnell et al. 2013) with default settings. Differentially expressed genes were

144 determined by pairwise comparison of a given condition to the other three conditions for a total

145 of six pairwise comparisons. Genes were considered significantly differentially expressed when 
146 they showed $a \geq 1.5 \log 2$ (fold change) in any of the conditions with a false discovery rate (FDR)-

147 corrected P value (q value) $\leq 0.05$ (Tables S3-S6). Principal component analysis was performed

148 with Canoco 5.0 (ter Braak \& Smilauer 2012) on log-transformed gene transcript abundances

149 using Hellinger standardization. Gene expression heatmaps were generated based on gene

150 transcript abundances using R v3.1.0 and R-packages svDialogs and gplots.

151 See the Supplemental Methods in Text S1 for details on growth on different carbohydrate media 152 and whole genome transcriptome analysis.

\section{Metagenomic investigations}

154 The datasets PRJNA237362 (Gevers et al., 2014) and PRJNA298762 (Alipour et al., 2016) were

155 analysed as relevant representative publicly available 16s rRNA gene amplicon datasets for the 156 presence of 16S rRNA gene sequences closely related to that of $R$. ilealis with NG-Tax version 1570.3 (Ramiro-Garcia et al., 2016) with the -classifyRatio argument set to 0.9.

\section{Nucleotide sequence accession number}

159 All related data have been deposited in the European Nucleotide Archive. The raw reads for the 160 genome of $R$. ilealis CRIB $^{\mathrm{T}}$ can be accessed via the accession numbers ERR366773, ERX397233, 161 ERX397242 and ERX339449. The assembly can be accessed under LN555523-LN555524. The 162 RNAseq data have been deposited under the numbers ERS533849- ERS533861.

\section{Results}

\section{Genome analysis}


165 Global genome features

166 R. ilealis $\mathrm{CRIB}^{\mathrm{T}}$ contains a single, circular chromosome of 2,581,778 bp and a plasmid of 6,145

167 bp (Table 1 and Fig. 1). The chromosome contains 2,351 predicted protein CDS, of which 321

168 were annotated as hypothetical and for 91, only a domain of unknown function could be assigned.

169 The plasmid carries eight predicted protein CDS, of which none was recognized for having a

170 metabolic or replicative function. Furthermore, it appears to be a non-mobilizable plasmid, given

171 that it lacks any known mobilization-associated genes. The overall $\mathrm{G}+\mathrm{C}$ content of the genome is

$17227.9 \%$, which is in good agreement with a $\mathrm{G}+\mathrm{C}$ content of $28.1 \mathrm{~mol} \%$ previously determined for

173 R. ilealis CRIB ${ }^{\mathrm{T}}$ by HPLC methods (Gerritsen et al. 2014).

174 INSERT TABLE 1

\section{INSERT FIGURE 1}

176 With a total of 14 copies of the $16 \mathrm{~S}$ ribosomal RNA (rRNA) gene, $R$. ilealis $\mathrm{CRIB}^{\mathrm{T}}$ is among the

177 species with the highest number of 16S rRNA gene copies reported up to this date (Lee et al.

178 2009). High numbers of rRNA operons have been proposed to be indicative for fast growth and to

179 allow microbes to respond quickly to changes in available resources (Klappenbach et al., 2000).

180 In addition, a high copy number of the rRNA operon has been suggested to be essential for

181 successful sporulation and germination (Yano et al. 2013). This is also reflected in the

182 observation that in general the species that contain the highest number of reported rRNA operons,

183 including $R$. ilealis $\mathrm{CRIB}^{\mathrm{T}}$, belong to the spore-forming bacterial orders Bacillales and

184 Clostridiales. Not all of the $16 \mathrm{~S}$ rRNA gene copies in R. ilealis $\mathrm{CRIB}^{\mathrm{T}}$ are embedded in the 185 conserved 16S-23S-5S rRNA operon structure. Of the fifteen locations containing rRNA genes, 186 ten are in the classical order 16S-23S-5S. The other five operons are characterized by duplicated 
187 or missing rRNA genes, or a different order of the genes. It should be noted that the current

188 assembly contains three gaps, all of which are located within rRNA operons. Diverging rRNA

189 operon structures have been reported for other genomes containing multiple rRNA operons, as a

190 result of duplications (Bensaadi-Merchermek et al. 1995; Schwartz et al. 1992).

191 A cluster of orthologous genes (COG) category (Tatusov et al., 1997) could be assigned to 1,647

192 of the predicted proteins (70\%) including 372 proteins (16\%) assigned to the categories $\mathrm{R}$

193 (general function prediction only) and S (function unknown) (Fig. S1). With InterProScan a

194 predicted function could be assigned to $82 \%$ of the predicted proteins. Based on the InterPro and

195 PRIAM classifications (Claudel-Renard et al. 2003), an enzymatic function could be predicted

196 for more than 500 proteins.

\section{General metabolic pathways}

198 Analysis of the CDS predicted from the R. ilealis $\mathrm{CRIB}^{\mathrm{T}}$ genome revealed the presence of a

199 complete set of enzymes for the glycolytic pathway. In line with the anaerobic lifestyle of the

200 organism, enzymes for the oxidative phase of the pentose phosphate pathway could not be

201 detected. Additionally, the genes that encode enzymes involved in the tricarboxylic acid cycle

202 were lacking. Subsequently a metabolic model was constructed with Pathway tools v18.0. A flux

203 balance analysis with the model was performed, suggesting that $R$. ilealis CRIB $^{\mathrm{T}}$ is a mixed acid

204 fermenter as previously reported (Gerritsen et al. 2014). Predicted end products of fermentation

205 are a mixture of acetate, formate, lactate and ethanol, with the possibility of gas formation $\left(\mathrm{CO}_{2}\right.$

206 and $\mathrm{H}_{2}$ ). In addition to ethanol, which can be produced during mixed acid fermentation, 1,2-

207 propanediol was predicted to be formed via the L-fucose degradation pathway. The fermentation

208 end products formate, acetate and lactate are predicted to be produced from pyruvate. No other

209 solvents were predicted to be produced by the metabolic model. The only metabolite produced by

210 R. ilealis $\mathrm{CRIB}^{\mathrm{T}}$ that was not accounted for by the metabolic model, was propionate. None of the 
211 three established pathways for propionate production in the intestinal tract, i.e. the succinate,

212 acrylate or the propanediol pathway (Reichardt et al. 2014), could be identified at the genetic

213 level in the genome of $R$. ilealis $\mathrm{CRIB}^{\mathrm{T}}$. Although propionate is only produced in low amounts

214 (max. $3 \mathrm{mM}$ in $24 \mathrm{~h}$ ) it is noteworthy because propionate production was observed repeatedly

215 during in vitro growth in this study (see Table 2) and as previously reported (Gerritsen et al.

216 2014).

217 The analysis of the genome and the prediction by the model indicated that fermentation is

218 probably the main process for energy conservation in $R$. ilealis. However, the presence of a

219 sulfite reductase gene cluster (CRIB_1284-CRIB_1286) of the dissimilatory asrC-type (Dhillon

220 et al. 2005) points at possible anaerobic respiration. Similar siroheme-dependent sulfite

221 reductases are found in many close-relatives of $R$. ilealis such as I. bartlettii, Clostridium

222 sordellii and C. difficile (Czyzewski \& Wang 2012). Sulfite reduction by R. ilealis CRIB $^{\mathrm{T}}$, and

223 close relatives, has been previously demonstrated in vitro (Gerritsen et al. 2014), and increased

224 growth yield and metabolite production was observed in the presence of sulfite for $R$. ilealis

225 CRIB $^{\mathrm{T}}$ (Table S7). In the intestinal tract, sulfite is derived from food sources that contain sulfite

226 as a preservative, and it has been shown that neutrophils release sulfite as a part of the host

227 defence against microbes (Mitsuhashi et al. 1998).

\section{Metabolism of growth factors and cofactors}

229 Complete pathways are present for the biosynthesis of the amino acids aspartate, asparagine,

230 glutamate, glutamine and cysteine, using carbon skeletons available from central metabolites or

231 via conversion of other amino acids. However, many genes encoding enzymes required for

232 biosynthesis of other amino acids appeared to be absent in $R$. ilealis $\mathrm{CRIB}^{\mathrm{T}}$. As most missing 233 genes are part of well-studied pathways, it is unlikely these functionalities are encoded by 234 unknown genes and likely represent true auxotrophies. The absence of genes to produce 
235 branched-chain amino acids (leucine, isoleucine and valine) was also reflected in the absence of

236 branched chain fatty acids in the cell membrane of $R$. ilealis, which is characteristic for the genus

237 Romboutsia (Gerritsen et al. 2014). From these observations it can be concluded that $R$. ilealis

238 depends on a number of exogenous amino acids, peptides and/or proteins to fuel protein

239 synthesis. The dependency on an exogenous source of amino acids is reflected by the

240 identification of multiple amino acid transporters, including an arginine/ornithine antiporter,

241 multiple serine/threonine exchangers, a transporter for branched amino acids, and several amino

242 acid symporters and permeases without a predicted specificity. Furthermore, numerous genes

243 were annotated as protease or peptidase, including several with a signal peptide.

244 R. ilealis $\mathrm{CRIB}^{\mathrm{T}}$ appears to contain all genes for de novo purine and pyrimidine synthesis, as well

245 as for the production of the coenzymes NAD and FAD via salvage pathways from niacin and

246 riboflavin, respectively. While some organic cofactors can be produced by $R$. ilealis CRIB $^{\mathrm{T}}$, it

247 mainly relies on salvage pathways (e.g. for lipoic acid) or exogenous sources for the supply of

248 precursors, mainly in the form of vitamins (e.g. thiamin, riboflavin, niacin, pantothenate,

249 pyridoxine, biotin, vitamin B12).

\section{Carbohydrate transport and metabolism}

251 As previously reported, $R$. ilealis $\mathrm{CRIB}^{\mathrm{T}}$ is able to utilize a wide variety of carbohydrates

252 (Gerritsen et al. 2014). Previously, good growth of $R$. ilealis on L-fucose, glucose, raffinose and

253 sucrose was described, in addition to moderate growth on D-arabinose and D-galactose and weak

254 growth on D-fructose, inulin, lactose, maltose and melibiose. Growth on L-fucose, fructose,

255 galactose, glucose, lactose, maltose, melibiose, raffinose and sucrose was predicted from the

256 genome-scale metabolic model as well. For these different carbohydrates, the genes encoding the

257 specific carbohydrate degradation enzymes were found distributed throughout the genome in

258 gene clusters together with their respective transporters and transcriptional regulator. The only 
259 carbohydrate utilized by $R$. ilealis $\mathrm{CRIB}^{\mathrm{T}}$ that was not predicted based on the metabolic model,

260 was D-arabinose. Although a separate arabinose transporter, similar to the maltose and sucrose

261 transporters, could be identified in the genome R. ilealis $\mathrm{CRIB}^{\mathrm{T}}$, no separate pathway for the use

262 of D-arabinose could be predicted, However, it is likely that the L-fucose degradation pathway

263 (encoded by genes CRIB_1294-CRIB_1298) is also used for D-arabinose utilization as is also

264 observed in other intestinal species (LeBlanc \& Mortlock 1971). In addition to the carbohydrates

265 for which growth was studied, a gene cluster involved in the degradation of the host-derived

266 carbohydrate sialic acid could be predicted (CRIB_613-CRIB_619) (Almagro-Moreno \& Boyd

267 2009). The structure of this gene cluster is similar to the one identified in C. difficile ( $\mathrm{Ng}$ et al.

268 2013). The ability to degrade the predominantly host-derived carbohydrates, L-fucose and sialic

269 acid, suggest a role in the utilization of mucin, an abundant host-derived glycoprotein in the

270 intestinal tract (Derrien et al. 2010; Ouwerkerk et al. 2013). However, no growth on mucin was

271 observed (Table S7), which is in line with the lack of a predicted extracellular fucosidase and/or

272 sialidase.

\section{Other genes encoding niche-specific functionalities}

274 A gene cluster encoding a urease, consisting of three subunits (ure $A B C$ ), and a number of urease

275 accessory genes was identified (CRIB_1381-CRIB_1388). The gene cluster identified in $R$.

276 ilealis $\mathrm{CRIB}^{\mathrm{T}}$ is very similar to the urease gene cluster in the genome of C. sordellii (Fig. S3), a

277 species in which the urease activity is used to phenotypically distinguish C. sordellii strains from

278 C. bifermentans strains (Roggentin et al. 1985). Furthermore, a possible ammonium transporter

279 (CRIB_1389) was identified in the genome of $R$. ilealis CRIB $^{\mathrm{T}}$ next to the urease gene cluster.

280 Ureases are nickel-containing metalloenzymes that catalyse the hydrolysis of urea to ammonia

281 and carbon dioxide, and thereby these enzymes allow microbes to use urea as nitrogen source by

282 assimilation via glutamate. They are ubiquitous proteins occurring in diverse organisms (Mobley 
283 et al. 1995). In the intestinal environment, where urea is abundantly present (Fuller \& Reeds

284 1998), some bacteria use ureases to survive the acidic conditions in the upper part of the intestinal

285 tract as urea hydrolysis leads to a local increase in pH (Rutherford 2014).

286 Another gene encoding a niche-specific functionality is the predicted choloylglycine hydrolase.

287 Proteins within the choloylglycine hydrolase family are bile salt hydrolases (BSHs), also known

288 as conjugated bile acid hydrolases (CBAHs), that are widespread among intestinal microbes

289 (Ridlon et al. 2006). They are involved in the hydrolysis of the amide linkage in conjugated bile

290 salts, releasing primary bile acids. There is a large heterogeneity among BSHs, for example with

291 respect to their substrate specificity. The BSH of $R$. ilealis CRIB $^{\mathrm{T}}$ was found to be the most

292 similar to the one found in Clostridium butyricum. Although the physiological advantages of

293 BSHs for the microbes are not completely understood, it has been hypothesized that they

294 constitute a mechanism to detoxify bile salts and thereby enhance bacterial colonization

295 (Czyzewski \& Wang 2012).

296 Metabolite and transcriptome analysis

297 Metabolite and transcriptome analysis of $R$. ilealis CRIB ${ }^{T}$ during growth on different 298 carbohydrates

299 To study key pathways predicted to be involved in carbohydrate utilization and their regulation in

300 more detail, a genome-wide transcriptome analysis was performed, focussing on four

301 experimental conditions. Firstly, growth on glucose, a preferred substrate for many microbes

302 present in the intestinal tract, was studied. Secondly the growth on fructans, oligo-and

303 polysaccharides present in many food items was examined. Previously weak growth on inulin, a

304 polysaccharide consisting of long chains of $ß 1 \square 2$ linked fructose units, was observed (Gerritsen

305 et al. 2014). For this study a shorter fructan (FOS P06, DP2-4) was chosen, because growth on 
306 shorter fructans is likely more relevant for microbes living in the small intestine (Zoetendal et al.

307 2012). Thirdly, growth on L-fucose was examined, as growth on this substrate was found to be

308 unique for $R$. ilealis $\mathrm{CRIB}^{\mathrm{T}}$ compared to other related microbes. Finally, $R$. ilealis $\mathrm{CRIB}^{\mathrm{T}}$ was

309 also grown in the basal medium in the absence of an additional carbon source for comparison

310 (control condition).

311 Based on measurements of optical density and $\mathrm{pH}$ during growth (growth characteristics of

312 individual cultures can be found in Table S1), samples were drawn in the mid-exponential phase

313 ( 8-10 h incubation; used for transcriptome analysis) and in stationary phase ( $24 \mathrm{~h}$ incubation),

314 and sugar utilization and fermentation products were measured with HPLC (Table 2). In neither

315 of the experimental conditions the supplied carbohydrates were depleted, and metabolites were

316 still produced at the time of sampling at $\sim 8-10 \mathrm{~h}$ and $24 \mathrm{~h}$, which further confirmed that samples

317 obtained for transcriptome analysis at $\sim 8-10 \mathrm{~h}$ were taken during exponential growth. In the FOS

318 cultures, an accumulation of extra-cellular fructose was observed. As predicted from the

319 metabolic model, growth on glucose resulted in the production of formate, acetate and lactate

320 (Table 2).

\section{INSERT TABLE 2}

322 Growth on FOS was marginally lower than that on glucose, however, after $24 \mathrm{~h}$ of growth, the

323 same fermentation products were observed in similar amounts (Table 2). Growth on L-fucose

324 showed production of 1,2-propanediol instead of lactate. The fact that 1,2-propanediol was

325 observed in one of the control cultures could be explained by the fact that an L-fucose grown

326 culture was used as inoculum for this culture, leading to carry-over of minor amounts of 327 metabolites. 
328 For the genome-wide transcriptome analysis of triplicate cultures grown in the four different

329 conditions (i.e. a total of 12 cultures), a total of 159,250,634 150bp-reads were generated by

330 RNA-seq (overview in Table S2). Principal component analysis of the transcriptomes of the

331 individual cultures showed that the cultures clustered by condition (Fig. 2).

332 INSERT FIGURE 2

333 Differential expression of genes involved in carbohydrate degradation and fermentation in $R$.

334 ilealis CRIB $^{T}$

335 To identify differentially regulated genes, pairwise comparisons were done with cuffdiff (Trapnell

336 et al. 2013) using a cut off of $\geq 1.5 \log 2$ (fold-change) and q-value $\leq 0.05$. Figure 3 shows a heat

337 map of all differentially regulated genes, and exact numbers can be found in Tables S3-6.

338 INSERT FIGURE 3

339 INSERT FIGURE 4

340 The gene cluster involved in glycolysis (CRIB_186-CRIB_191) was most abundantly expressed

341 in the conditions that support the highest growth rates determined by the highest cell density

342 reached in the time period that was measured (glucose, followed by FOS; Fig. 3). This was also

343 reflected in the fact that expression of genes encoding proteins involved in replication such as

344 ribosomal proteins, proteins involved in cell wall biosynthesis and general cell division processes

345 were most strongly expressed during growth in the presence of glucose and to a lesser extent

346 FOS. Other genes involved in the central sugar metabolic pathways (e.g. CRIB_1849, CRIB_140,

347 CRIB_2223, and CRIB_105) were upregulated in these conditions, albeit not significantly 
348 differentially regulated. This suggests that these genes are less tightly regulated at the

349 transcriptional level, probably because they are also involved in other processes than sugar

350 degradation (Commichau et al. 2009). The metabolic model suggests that this is indeed the case,

351 as some of the enzymes produce intermediates which can be consumed by fatty acid biosynthesis

352 and amino acid biosynthesis processes.

353 Altogether, the transcriptome of $R$. ilealis $\mathrm{CRIB}^{\mathrm{T}}$ grown on FOS was very similar to its

354 transcriptome when grown on glucose (Fig. 2), with only 18 genes significantly upregulated

355 during growth in the presence of FOS compared to glucose (Table S4). Apparent was the

356 upregulation of the gene clusters that code for proteins involved in the transport and degradation

357 of the respective sugars or their derivatives (Fig. 3). In the presence of glucose, the glucose-

358 specific PTS system (CIRB_2017-CRIB_2018) was significantly upregulated, together with its

359 associated transcriptional regulator (CRIB_2019). In turn, in the presence of FOS, two clusters

360 predicted to be involved in sucrose degradation (CRIB_148-CRIB_152 and CRIB_1458-1461)

361 were significantly upregulated. The third gene cluster predicted to be involved in sucrose

362 degradation (CRIB_1399-1400) was not significantly regulated during growth on FOS. However,

363 it should be noted that these genes are located in a cluster functionally annotated to melibiose

364 metabolism and are most likely regulated by the transcriptional regulator in this cluster. In

365 addition to the two sucrose degradation clusters, a transport cluster of unknown function

366 (CRIB_1506-CRIB_1509) was upregulated during growth on FOS, albeit only significantly when

367 compared to growth on glucose. During growth in the presence of L-fucose, the gene cluster

368 predicted to be involved in L-fucose degradation (CRIB_1294-CRIB_1298) was significantly

369 upregulated, including the gene encoding the corresponding transcriptional regulator

370 (CRIB_1299). An overview of the main carbohydrate degradation pathways regulated in the

371 different conditions is given in Figure 4. 
372 During growth on glucose, L-lactate dehydrogenase (CRIB_684) was significantly upregulated,

373 albeit not significantly compared to growth on FOS. This enzyme catalyses the reduction of

374 pyruvate resulting in the production of L-lactate and the reoxidation of the NADH formed during

375 glycolysis. Only at the time point of $24 \mathrm{~h}$, lactate was observed (Table 2). This suggests that at

376 time point $\sim 8-10 \mathrm{~h}$ the cells were starting to regenerate NAD by upregulating this gene. In the

377 presence of L-fucose, $\mathrm{NAD}^{+}$regeneration is achieved via the reduction of lactaldehyde to 1,2-

378 propanediol by lactaldehyde reductase (CRIB_1300), which was upregulated in the presence of

379 L-fucose together with the L-fucose degradation gene cluster. In the spent medium of L-fucose

380 grown cells, 1,2-propanediol was already seen at time point $\sim 8-10 \mathrm{~h}$, whereas no lactate

381 production was observed. Another way to regenerate $\mathrm{NAD}^{+}$is to reduce pyruvate to ethanol (Fig.

382 4). In the presence of both glucose and FOS, an upregulation was seen for the gene encoding the

383 bifunctional aldehyde/alcohol dehydrogenase (CRIB_2231), which converts acetyl-CoA to

384 ethanol. However, in none of the samples, ethanol was measured by HPLC analysis.

385 During growth on FOS, a small gene cluster (CRIB_601-CRIB_603) that includes a gene 386 encoding an alternative sigma factor was significantly upregulated. This was also apparent in the

387 control culture that was inoculated with FOS-preconditioned cells. This suggests that in the 388 presence of FOS (or its derivatives sucrose or fructose) transcription is also regulated by RNA 389 polymerase promoter recognition.

\section{Expression and regulation of other environmentally relevant functions in $R$. ilealis CRIB ${ }^{T}$}

391 Noteworthy was the significant upregulation of a gene cluster related to iron transport

392 (CRIB_892-CRIB-898) during growth on glucose and FOS compared to growth on L-fucose. The

393 significance of this gene cluster for carbohydrate utilization is not known, however, several

394 enzymes could be identified in the genome of R. ilealis $\mathrm{CRIB}^{\mathrm{T}}$ that use different forms of iron as

395 cofactor, for example the hydrogenases involved in hydrogen metabolism (Calusinska et al. 
396 2010), several ferredoxins, and the L-threonine dehydratase (CRIB-426) that was significantly

397 upregulated during growth on L-fucose. As multiple transporters involved in the transport of iron

398 compounds were predicted, it is possible that the uptake of iron provides a competitive advantage

399 to other microbes that are dependent on iron for respiration and other metabolic processes

400 (Kortman et al. 2014).

\section{Prevalence of $\boldsymbol{R}$. ilealis in human datasets}

402 R. ilealis was found to be a natural and abundant inhabitant of the rat small intestine, specifically

403 of the ileum (Gerritsen et al. 2011b). To study its prevalence in humans, 16S rRNA amplicon

404 sequencing datasets were investigated for the presence of $R$. ilealis-like 16S rRNA gene

405 sequences. Unfortunately, with respect to composition analysis of human ileum samples, there is

406 only a limited number of datasets available due to the sampling difficulties that are the result of

407 the inaccessibility of this part of the intestinal tract. In the dataset published by Alipour et al.

408 (2016), a paediatric human dataset with samples from both healthy individuals and inflammatory

409 bowel disease patients, we were not able to identify any Romboutsia-like 16S rRNA gene

410 sequences. In the dataset published by Gevers et al. (2014), one of the biggest 16S rRNA gene

411 datasets published to date that includes samples obtained from multiple gastrointestinal locations

412 (ileal and rectal biopsies and faecal samples) from both healthy individuals and inflammatory

413 bowel disease patients, only a limited number of R. ilealis-like 16S rRNA gene sequences could

414 be identified. In this dataset, the genus Romboutsia could be identified in two samples, with a

415 relative abundance of $0.1 \%$ and $0.2 \%$. In 173 cases the family Peptostreptococcaceae could be

416 identified in these samples, but it was not possible to differentiate between the genera

417 Romboutsia and Intestinibacter, due to $100 \%$ identity of their rRNA gene sequences in this

418 region. The Peptostreptococcaceae-positive samples were obtained from both healthy and

419 diseased individuals (including at least one with ileal overgrowth and a Peptostreptococcaceae 
420 abundance of $46 \%$ ). It should be noted that both datasets contain only sequence data from

421 paediatric ileal biopsy samples and therefore only mucosa-associated microbiota could be studied

422 limited to a human population $<17$ years of age, which could explain the low prevalence of $R$.

423 ilealis-like 16S rRNA gene sequences. Unfortunately, due to the limited number of available

424 human datasets and the low prevalence of $R$. ilealis-like 16S rRNA gene sequences in ileal biopsy

425 samples, it was not possible to find positive or negative correlations between prevalence and/or

426 abundance of $R$. ilealis and specific human diseases.

\section{Discussion}

428 Gerritsen et al. (2011b) have shown by 16S rRNA gene sequence-based analysis that $R$. ilealis

$429 \mathrm{CRIB}^{\mathrm{T}}$ is a dominant member of the small intestine microbiota in rats, especially in the ileum.

430 The genomic and transcriptomic analysis of $R$. ilealis $\mathrm{CRIB}^{\mathrm{T}}$ reported here provides new insights

431 into the genetic and functional potential of this inhabitant of the small intestine. Genomic analysis

432 revealed the presence of metabolic pathways for the utilization of a wide array of simple

433 carbohydrates in addition to a multitude of carbohydrate uptake systems that included a series of

434 PTS systems, carbohydrate specific ABC transporters, permeases and symporters. This is in

435 agreement with prior observations by Zoetendal et al. (2012), who reported that the small

436 intestinal microbiome is enriched for genes involved in the consumption of simple carbohydrates.

437 However, small disagreements with prior observations were also observed. An enrichment for

438 amino acid metabolism (Zoetendal et al., 2012) was not visible in $R$. ilealis $\mathrm{CRIB}^{\mathrm{T}}$, and

439 considerable less COGs could be classified than for the average small intestinal bacterium

440 (Leimena et al., 2013).

441 Since the small intestine is an environment in which environmental conditions change quickly

442 due to the varying food intake of the host, microorganisms in this environment must be able to 443 respond rapidly to such changes. As previously mentioned, the high number of rRNA operons 
444 found in the genome of $R$. ilealis $\mathrm{CRIB}^{\mathrm{T}}$ is an indication that this this strain is indeed able to

445 adapt its metabolism quickly in response to changing conditions, as a high rRNA copy number

446 has been associated with this trait (Klappenbach et al., 2000). Considering the small intestinal

447 habitat, we chose to focus on key pathways involved in the utilization of specific diet- and host-

448 derived carbon sources by whole-genome transcriptome analysis.

\section{Degradation of FOS and its possible role in cross-feeding}

450 In the intestinal tract, the diet-derived carbohydrates that the host is unable to digest are important

451 sources of energy for many microbes. In return, the host is dependent on the degradation of food-

452 derived indigestible component by microbes for the release of certain essential metabolites (e.g.

453 SCFA). Here we examined the growth of $R$. ilealis $\mathrm{CRIB}^{\mathrm{T}}$ on FOS, a relatively simple

454 oligosaccharide that is indigestible by the host, and the metabolites that were released. The

455 transcriptome of $R$. ilealis $\mathrm{CRIB}^{\mathrm{T}}$ grown on FOS was very similar to its transcriptome when

456 grown on glucose, a monosaccharide used by the majority of microbes present in the intestinal

457 tract. This is not surprising considering that glucose in addition to fructose is one of the two

458 subunits present in FOS. Noteworthy was the accumulation of fructose in the culture supernatant

459 during growth of $R$. ilealis $\mathrm{CRIB}^{\mathrm{T}}$ on FOS. Based on the genomic analysis there are no apparent

460 reasons why fructose should not be metabolized as all the necessary metabolic enzymes are

461 present. However, it has been previously observed that $R$. ilealis only grows weakly on D-

462 fructose (Gerritsen et al. 2014). The absence of a fructose-specific transporter, which could be

463 identified in close relatives that are able to grow on D-fructose, might explain the fructose

464 accumulation during growth of $R$. ilealis $\mathrm{CRIB}^{\mathrm{T}}$ on FOS.

465 Differential gene expression analysis demonstrated the apparent FOS-induced upregulation of

466 two separate gene clusters that were predicted to be involved in sucrose transport and

467 degradation. However, based on the genomic analysis no apparent pathways could be identified 
468 to be responsible for FOS degradation. A simple explanation for the observed growth on FOS

469 could be extracellular degradation of FOS, followed by import of sucrose and/or glucose into the

470 cell. Fructan degradation by extracellular enzymes is described for other (intestinal) microbes

471 (van Hijum et al. 2006). The observed accumulation of fructose during growth of $R$. ilealis

$472 \mathrm{CRIB}^{\mathrm{T}}$ on FOS supports the hypothesis of extracellular degradation. However, no extracellular

473 fructansucrase or glucansucrase could be predicted. Furthermore, no new candidates for this

474 activity could be identified via the differential gene expression analysis described here. However,

475 one possible candidate could be the predicted beta-fructofuranosidase present in the PTS system-

476 containing sucrose degradation gene cluster. Next to the beta-fructofuranosidase-encoding gene, a

477 gene was found to which no function could be assigned, but that was predicted to have a

478 transmembrane region and a domain which could be involved in transport. Given that both loci

479 overlap by a few nucleotides, and that the overlap is within a homopolymer region, it is possible

480 that both loci form one protein due to ribosomal slippage on the homopolymer (Sharma et al.

481 2014). This could possibly lead to an external membrane-bound enzymatically active protein,

482 which would explain the accumulation of fructose. Future studies with mutant strains might shed

483 more light on the specific contribution of the two predicted sucrose degradation gene clusters to

484 the degradation of FOS, or even longer fructans (e.g. inulin), by R. ilealis $\mathrm{CRIB}^{\mathrm{T}}$. Altogether,

485 these results might indicate a possible role for $R$. ilealis $\mathrm{CRIB}^{\mathrm{T}}$ in intestinal cross-feeding

486 networks by releasing D-fructose during growth on fructans like FOS, which can function as

487 growth substrate for other microbes or be directly absorbed by the host.

\section{Fucose degradation and its advantages}

489 Besides diet-derived carbohydrates, also host-derived carbohydrates are an important source of 490 energy for some microbes. Unlike other members of the family Peptostreptococcaceae, R. ilealis

491 CRIB $^{\mathrm{T}}$ is able to grow on L-fucose, a predominantly host-derived carbon source (Gerritsen et al. 
492 2014). The transcriptome analysis confirmed the presence of a functional L-fucose degradation

493 pathway, similar to the pathways previously identified in other intestinal inhabitants such as $E$.

494 coli (Baldoma \& Aguilar 1988), Bacteroides thetaiotaomicron (Hooper et al. 1999) and

495 Roseburia inulinivorans (Scott et al. 2006). By gene sequence homology a similar pathway was

496 found in Clostridium perfringens and the more closely related C. sordellii (Fig. S2). L-fucose is a

497 common sugar present within the intestinal environment, since it is a monosaccharide that is an

498 abundant component of many N- and O-linked glycans and glycolipids produced by mammalian

499 cells, including the fucosylated glycans that are found at the terminal positions of mucin

500 glycoproteins (Becker \& Lowe 2003). Fucosylated mucin glycoproteins are especially found in

501 the (human) ileum (Robbe et al. 2004; Robbe et al. 2003). For both intestinal commensals and

502 pathogens the ability to utilize L-fucose has been demonstrated to provide a competitive

503 advantage in the intestinal environment (Hooper et al. 1999; Stahl et al. 2011). In R. ilealis, all

504 enzymes for L-fucose degradation are present in one cluster, however, no fucosidase-encoding

505 gene could be identified, which means that $R$. ilealis is not able to release L-fucose units from

506 fucosylated glycans (e.g. mucin) by itself. Hence, in the intestinal environment $R$. ilealis is

507 dependent on free L-fucose monosaccharides released by other microbes. Furthermore, a gene

508 cluster involved in degradation of sialic acid (Almagro-Moreno \& Boyd 2009; Vimr 2013; Vimr

509 et al. 2004) was predicted from the genome, but no extracellular sialidase could be identified,

510 which is similar to what has been found for C. difficile ( $\mathrm{Ng}$ et al. 2013). This suggests that also

511 for sialic acid, a common residue found in mucin glycoproteins, $R$. ilealis CRIB $^{\mathrm{T}}$ seems to be

512 dependent on the activity of other microbes. However, this also suggests that by its ability to use

513 L-fucose and sialic acid monosaccharides, $R$. ilealis $\mathrm{CRIB}^{\mathrm{T}}$ is dependent for these host-derived

514 sugars that are released by the action of extracellular enzymes of with mucus-degrading microbes

515 like B. thetaiotaomicron or Akkermansia muciniphila. Besides niche competition with other

516 commensals, fucose utilization may also be important in niche competition with pathogens. It 
517 was recently suggested that the host is able to regulate fucosylation of its intestinal epithelial cells

518 in response to pathogen-induced stress and that microbes that are able to use fucose as an energy

519 source may contribute to the protection of the host against infections by endogenous pathogens

520 (Pickard et al. 2014).

\section{Regulation of carbohydrate catabolism}

522 In the intestinal environment $R$. ilealis $\mathrm{CRIB}^{\mathrm{T}}$ will encounter a wide array of carbohydrates that

523 are either continually or transiently present. Prioritization of carbohydrate utilization is partly

524 achieved at the transcriptional level by the selective expression of genes. The primary mechanism

525 by which bacteria regulate the utilization of non-preferred carbohydrates in the presence of

526 preferred carbon sources is known as carbon catabolite repression (CCR), a hierarchical system

527 for coordinating sugar metabolism (Deutscher 2008). The fact that, compared to glucose and

528 FOS, L-fucose is utilized by a pathway that does not directly involve fructose-1,6-bisphosphate, a

529 key metabolite in the regulation of CCR of Gram-positive bacteria, made it possible to study

530 CCR by either glucose or FOS. The transcriptome analysis suggests that some genes and operons

531 in $R$. ilealis $\mathrm{CRIB}^{\mathrm{T}}$ were indeed subject to $\mathrm{CCR}$ in response to the presence of glucose. For

532 example, two gene clusters predicted to be involved in hexuronate metabolism (CRIB_649-

533 CRIB_652 and CRIB_2244-CRIB_2249), pathways that make the use of D-glucuronate and D-

534 galacturonates as sole carbon source possible, were significantly upregulated during growth in the

535 presence of L-fucose compared to growth on glucose (Table S5). In addition, the gene cluster

536 predicted to be involved in sialic acid utilization (CRIB_613-CRIB_616) was downregulated in

537 the presence of glucose as well. Furthermore, when comparing the expression of the gene cluster

538 involved in L-fucose degradation during growth on glucose relative to the growth in the absence

539 of a carbon source (control condition), this gene cluster appeared to be under CCR as well, in the 
540 presence of glucose (Table S5). These results suggest that in $R$. ilealis $\mathrm{CRIB}^{\mathrm{T}}$, multiple gene

541 clusters that are involved in the use of alternative carbon sources are subject to CCR.

\section{Expression and regulation of niche-specific functionalities in R. ilealis CRIB}

543 Microbes residing in the intestinal tract have to withstand the harsh environmental conditions

544 specific for the intestine. In this context, it was interesting that we identified a urease gene cluster

545 in R. ilealis CRIB $^{\mathrm{T}}$ (CRIB_1381-CRIB_1388), expression of which appeared to be induced in

546 carbon source limiting circumstances. The fact that this gene cluster was significantly

547 upregulated when grown in the absence of an additional carbon source compared to growth on

548 glucose, possibly suggests CCR of the urease gene cluster. However, upregulation of this gene

549 cluster in the absence of an exogenous carbon source might also be a possible mechanism. Urea

550 in the intestinal tract is derived from the breakdown of amino acids. Helicobacter pylori is a well-

551 known example where urease activity contributes to the survival of the bacterium in the acidic

552 environment of the stomach (Marshall et al. 1990). For some of the urease-positive bacteria, this

553 enzyme has been shown to act as a virulence factor as it is responsible for urea hydrolysis that

554 leads to increased $\mathrm{pH}$ and ammonia toxicity (Rutherford 2014). However, for commensal

555 intestinal bacteria ureases can probably function as colonization factors as well, as they

556 contribute in general to acid resistance and thereby play a role in gastrointestinal survival

557 (Marshall et al. 1990). Urea is released into all parts of the intestinal tract via diffusion from the

558 blood, but it has been reported that pancreatic excretions and bile are a main route of entry

559 (Bergner et al. 1986). So far, we have not been able to demonstrate urease activity in R. ilealis

$560 \mathrm{CRIB}^{\mathrm{T}}$ (Gerritsen et al. 2014). However, different mechanisms for the expression of urease have

561 been identified in other microbes: constitutive, inducible by urea, or controlled by nitrogen source

562 availability (Mobley et al. 1995). For C. perfringens for example, the urease activity, which is

563 plasmid borne, was shown to be only expressed in nitrogen-limiting conditions (Dupuy et al. 
564 1997). The increased urease gene expression by $R$. ilealis CRIB $^{\mathrm{T}}$ observed in the control

565 condition, in the absence of an additional carbohydrate, suggests an alternative mechanism for

566 regulation of urease gene expression.

\section{Conclusions}

568 We are just starting to elucidate the composition and function of the microbial communities in the

569 mammalian small intestine. Recently we have reported the isolation and characterization of $R$.

570 ilealis $\mathrm{CRIB}^{\mathrm{T}}$ from the small intestine of a rat (Gerritsen et al. 2014). In rats, this species was

571 identified to be a dominant member of the ileal microbiota (Gerritsen et al. 2011b). Here we

572 applied a holistic systems biology approach, involving several fields of experimental and

573 theoretical biology, to study $R$. ilealis $\mathrm{CRIB}^{\mathrm{T}}$. In conclusion, $R$. ilealis $\mathrm{CRIB}^{\mathrm{T}}$ is a strain that is

574 able to utilize an array of carbohydrates using different and partially redundant pathways. Its

575 ability to use host-derived sugars that are liberated by other microbes suggests that $R$. ilealis

$576 \mathrm{CRIB}^{\mathrm{T}}$ is dependent on mucus-degrading microbes, like B. thetaiotaomicron or A. muciniphila. In

577 contrast, it has only limited ability to de novo synthesize amino acids and vitamins, and hence the

578 organism shows an adaption to a nutrient-rich environment in which carbohydrates and

579 exogenous sources of amino acids and vitamins are abundantly available. In addition, we were

580 able to pinpoint potential mechanisms that might enable this organism to survive in the

581 competitive small intestinal environment. These mechanisms include bile salt hydrolase and

582 urease enzymes, which enhance the organism's ability to handle in particular small-intestinal

583 conditions.

584 It has to be emphasized that the results presented in this study correspond to one specific strain

585 and that different strains belonging to the same species could possibly encode for different

586 functions, including utilisation of specific glycans as previously described by Crost et al. (2013).

587 However, a deeper investigation of key players in the intestinal tract like $R$. ilealis CRIB $^{\mathrm{T}}$ and 
588 others will lead to a better understanding of how the microbial communities in us function as a

589 whole. The more we understand how each organism works, and how they interact, the better we

590 get an insight into these environments and can predict how nutrition will influence our health and

591 well-being.

\section{Acknowledgements}

593 The authors thank Hans Heilig (Wageningen University, Laboratory of Microbiology) for his help

594 with genomic DNA isolations and Jasper Koehorst (Wageningen University, Laboratory of

595 Systems and Synthetic Biology) for his help with the genome annotation. 
596 References

597 Alipour M, Zaidi D, Valcheva R, Jovel J, Martínez I, Sergi C, Walter J, Mason AL,

598 Wong GK, Dieleman LA, Carroll MW, Huynh HQ, and Wine E. 2016. Mucosal Barrier

599 Depletion and Loss of Bacterial Diversity are Primary Abnormalities in Paediatric

$600 \quad$ Ulcerative Colitis. Journal of Crohn's and Colitis, 462-471

601 Almagro-Moreno S, and Boyd EF. 2009. Insights into the evolution of sialic acid catabolism $602 \quad$ among bacteria. BMC Evol Biol 9:118.

603 Backhed F, Ley RE, Sonnenburg JL, Peterson DA, and Gordon JI. 2005. Host-bacterial 604

605

606

607

608

609

610

611

612

613

614

615

616

617

618

619

620

621

622

623

624

625

626

627

628

629

630

631

632

633

634

635

636

637

638

639

640

641

642

643

644

645

646

647

648

649 mutualism in the human intestine. Science 307:1915-1920.

Baldoma L, and Aguilar J. 1988. Metabolism of L-fucose and L-rhamnose in Escherichia coli: aerobic-anaerobic regulation of L-lactaldehyde dissimilation. Journal of bacteriology 170:416-421.

Becker DJ, and Lowe JB. 2003. Fucose: biosynthesis and biological function in mammals. Glycobiology 13:41R-53R.

Bensaadi-Merchermek N, Salvado JC, Cagnon C, Karama S, and Mouches C. 1995. Characterization of the unlinked 16S rDNA and 23S-5S rRNA operon of Wolbachia pipientis, a prokaryotic parasite of insect gonads. Gene 165:81-86.

Bergner H, Simon O, Zebrowska T, and Munchmeyer R. 1986. Studies on the secretion of amino acids and of urea into the gastrointestinal tract of pigs. 3. Secretion of urea determined by continuous intravenous infusion of 15N-urea. Arch Tierernahr 36:479-490.

Bland C, Ramsey TL, Sabree F, Lowe M, Brown K, Kyrpides NC, and Hugenholtz P. 2007. CRISPR recognition tool (CRT): a tool for automatic detection of clustered regularly interspaced palindromic repeats. BMC Bioinformatics 8:209.

Booijink CC, Zoetendal EG, Kleerebezem M, and de Vos WM. 2007. Microbial communities in the human small intestine: coupling diversity to metagenomics. Future microbiology 2:285-295.

Burge SW, Daub J, Eberhardt R, Tate J, Barquist L, Nawrocki EP, Eddy SR, Gardner PP, and Bateman A. 2013. Rfam 11.0: 10 years of RNA families. Nucleic Acids Res 41:D226-232.

Calusinska M, Happe T, Joris B, and Wilmotte A. 2010. The surprising diversity of clostridial hydrogenases: a comparative genomic perspective. Microbiology 156:1575-1588.

Claudel-Renard C, Chevalet C, Faraut T, and Kahn D. 2003. Enzyme-specific profiles for genome annotation: PRIAM. Nucleic Acids Res 31:6633-6639.

Commichau FM, Rothe FM, Herzberg C, Wagner E, Hellwig D, Lehnik-Habrink M, Hammer E, Volker U, and Stulke J. 2009. Novel activities of glycolytic enzymes in Bacillus subtilis: interactions with essential proteins involved in mRNA processing. Mol Cell Proteomics $8: 1350-1360$.

Crost EH, Tailford LE, Le Gall G, Fons M, Henrissat B, Juge N. 2013. Utilisation of mucin glycans by the human gut symbiont Ruminococcus gnavus is strain-dependent. PloS one 8:e76341.

Czyzewski BK, and Wang DN. 2012. Identification and characterization of a bacterial hydrosulphide ion channel. Nature 483:494-497.

Derrien M, van Passel MW, van de Bovenkamp JH, Schipper RG, de Vos WM, and Dekker J. 2010. Mucin-bacterial interactions in the human oral cavity and digestive tract. Gut Microbes 1:254-268.

Deutscher J. 2008. The mechanisms of carbon catabolite repression in bacteria. Curr Opin Microbiol 11:87-93.

Dhillon A, Goswami S, Riley M, Teske A, and Sogin M. 2005. Domain evolution and functional diversification of sulfite reductases. Astrobiology 5:18-29.

Dupuy B, Daube G, Popoff MR, and Cole ST. 1997. Clostridium perfringens urease genes are plasmid borne. Infection and immunity 65:2313-2320.

Elia M, and Cummings JH. 2007. Physiological aspects of energy metabolism and gastrointestinal effects of carbohydrates. Eur J Clin Nutr 61 Suppl 1:S40-74.

Flint HJ, Scott KP, Duncan SH, Louis P, and Forano E. 2012. Microbial degradation of complex carbohydrates in the gut. Gut Microbes 3:289-306. 
650 Fuller MF, and Reeds PJ. 1998. Nitrogen cycling in the gut. Annu Rev Nutr 18:385-411.

651 Galperin MY, Brover V, Tolstoy I, and Yutin N. 2016. Phylogenomic analysis of the family

652

653

654

655

656

657

658

659

660

661

662

663

664

665

666

667

668

669

670

671

672

673

674

675

676

677

678

679

680

681

682

683

684

685

686

687

688

689

690

691

692

693

694

695

696

697

698

699

700

701

702

703 Peptostreptococcaceae (Clostridium cluster XI) and proposal for reclassification of Clostridium litorale (Fendrich et al. 1991) and Eubacterium acidaminophilum (Zindel et al. 1989) as Peptoclostridium litorale gen. nov. comb. nov. and Peptoclostridium acidaminophilum comb. nov. International journal of systematic and evolutionary microbiology 66: 5506-5513

Gerritsen J, Fuentes S, Grievink W, van Niftrik L, Tindall BJ, Timmerman HM, Rijkers GT, and Smidt H. 2014. Characterization of Romboutsia ilealis gen. nov., sp. nov., isolated from the gastro-intestinal tract of a rat, and proposal for the reclassification of five closely related members of the genus Clostridium into the genera Romboutsia gen. nov., Intestinibacter gen. nov., Terrisporobacter gen. nov. and Asaccharospora gen. nov. International journal of systematic and evolutionary microbiology 64:1600-1616.

Gerritsen J, Smidt H, Rijkers GT, and de Vos WM. 2011a. Intestinal microbiota in human health and disease: the impact of probiotics. Genes Nutr 6:209-240.

Gerritsen J, Timmerman HM, Fuentes S, van Minnen LP, Panneman H, Konstantinov SR, Rombouts FM, Gooszen HG, Akkermans LM, Smidt H, and Rijkers GT. 2011b. Correlation between protection against sepsis by probiotic therapy and stimulation of a novel bacterial phylotype. Applied and environmental microbiology 77:7749-7756.

Gevers D, Kugathasan S, Denson LA, Vazquez-Baeza Y, van Treuren W,Ren B, Schwager E, Knights D, Song SJ, Yassour M, Morgan XC, Kostic AD, Luo C, Gonzalez A, McDonald D, Haberman Y, Walters T, Baker S, Rosh J, Stephens M, Heyman M, Markowitz J, Baldassano R, Griffiths A, Sylvester F, Mack D, Kim S, Crandall W, Hyams J, Huttenhower C, Knight R and Xavier RJ. 2014. The Treatment-Naive Microbiome in New-Onset Crohn's Disease. Cell Host \& Microbe 15, 382-392

Heinken A, Sahoo S, Fleming RM, and Thiele I. 2013. Systems-level characterization of a hostmicrobe metabolic symbiosis in the mammalian gut. Gut Microbes 4:28-40.

Hooper LV, Xu J, Falk PG, Midtvedt T, and Gordon JI. 1999. A molecular sensor that allows a gut commensal to control its nutrient foundation in a competitive ecosystem. Proc Natl Acad Sci U S A 96:9833-9838.

Hunter S, Jones P, Mitchell A, Apweiler R, Attwood TK, Bateman A, Bernard T, Binns D, Bork P, Burge S, de Castro E, Coggill P, Corbett M, Das U, Daugherty L, Duquenne L, Finn RD, Fraser M, Gough J, Haft D, Hulo N, Kahn D, Kelly E, Letunic I, Lonsdale D, Lopez R, Madera M, Maslen J, McAnulla C, McDowall J, McMenamin C, Mi H, MutowoMuellenet P, Mulder N, Natale D, Orengo C, Pesseat S, Punta M, Quinn AF, Rivoire C, Sangrador-Vegas A, Selengut JD, Sigrist CJ, Scheremetjew M, Tate J, Thimmajanarthanan M, Thomas PD, Wu CH, Yeats C, and Yong SY. 2012. InterPro in 2011: new developments in the family and domain prediction database. Nucleic Acids Res 40:D306312.

Hyatt D, Chen GL, Locascio PF, Land ML, Larimer FW, and Hauser LJ. 2010. Prodigal: prokaryotic gene recognition and translation initiation site identification. $B M C$ Bioinformatics 11:119.

Ingram-Smith C, Gorrell A, Lawrence SH, Iyer P, Smith K, and Ferry JG. 2005. Characterization of the acetate binding pocket in the Methanosarcina thermophila acetate kinase. Journal of bacteriology 187:2386-2394.

Karasawa T, Ikoma S, Yamakawa K, and Nakamura S. 1995. A defined growth medium for clostridium difficile. Microbiology 141:371-375.

Klappenbach, JA, Dunbar, JM, Schmidt, TM. 2000. rRNA operon copy number reflects ecological strategies of bacteria. Applied and environmental microbiology 66:1328-1333

Kortman GA, Raffatellu M, Swinkels DW, and Tjalsma H. 2014. Nutritional iron turned inside out: intestinal stress from a gut microbial perspective. FEMS Microbiol Rev.

Lagesen K, Hallin P, Rodland EA, Staerfeldt HH, Rognes T, and Ussery DW. 2007. RNAmmer: consistent and rapid annotation of ribosomal RNA genes. Nucleic Acids Res 35:31003108 . 
704 Lange K, Hugenholtz F, Schols H, Kleerebezem M, Smidt H, Müller M, and Hooiveld GJEJ.

705

706

707

708

709

710

711

712

713

714

715

716

717

718

719

720

721

722

723

724

725

726

727

728

729

730

731

732

733

734

735

736

737

738

739

740

741

742

743

744

745

746

747

748

749

750

751

752

753

754

755

756

757

758
2015. Comparison of the effects of five dietary fibers on mucosal transcriptional profiles and luminial micribiota composition and SCFA concentrations in murine colon. Molecular Nutrition \& Food Research in press.

Langmead B, and Salzberg SL. 2012. Fast gapped-read alignment with Bowtie 2. Nat Methods 9:357-359.

Latendresse M, Krummenacker M, Trupp M, Karp PD. 2012. Construction and completion of flux balance models from pathway databases. Bioinformatics 28:388-396.

LeBlanc DJ, and Mortlock RP. 1971. Metabolism of D-arabinose: a new pathway in Escherichia coli. Journal of bacteriology 106:90-96.

Lee ZM, Bussema C, 3rd, and Schmidt TM. 2009. rrnDB: documenting the number of rRNA and tRNA genes in bacteria and archaea. Nucleic Acids Res 37:D489-493.

Leimena MM, Ramiro-Garcia J, Davids M, Van den Bogert B, Smidt H, Smid EJ, Boekhorst J, Zoetendal EG, Schaap PJ, and Kleerebezem M. 2013. A comprehensive metatranscriptome analysis pipeline and its validation using human small intestine microbiota datasets. BMC Genomics 14.

Li M, Wang B, Zhang M, Rantalainen M, Wang S, Zhou H, Zhang Y, Shen J, Pang X, Wei H, Chen Y, Lu H, Zuo J, Su M, Qiu Y, Jia W, Xiao C, Smith LM, Yang S, Holmes E, Tang H, Zhao G, Nicholson JK, Li L, and Zhao L. 2008. Symbiotic gut microbes modulate human metabolic phenotypes. Proc Natl Acad Sci U S A 105:2117-2122.

Lowe TM, and Eddy SR. 1997. tRNAscan-SE: a program for improved detection of transfer RNA genes in genomic sequence. Nucleic Acids Res 25:955-964.

Marshall BJ, Barrett LJ, Prakash C, McCallum RW, and Guerrant RL. 1990. Urea protects Helicobacter (Campylobacter) pylori from the bactericidal effect of acid. Gastroenterology 99:697-702.

Mitsuhashi H, Nojima Y, Tanaka T, Ueki K, Maezawa A, Yano S, and Naruse T. 1998. Sulfite is released by human neutrophils in response to stimulation with lipopolysaccharide. $J$ Leukoc Biol 64:595-599.

Mobley HL, Island MD, and Hausinger RP. 1995. Molecular biology of microbial ureases. Microbiol Rev 59:451-480.

Ng KM, Ferreyra JA, Higginbottom SK, Lynch JB, Kashyap PC, Gopinath S, Naidu N, Choudhury B, Weimer BC, Monack DM, and Sonnenburg JL. 2013. Microbiota-liberated host sugars facilitate post-antibiotic expansion of enteric pathogens. Nature 502:96-99.

Notebaart RA, Szappanos B, Kintses B, Pal F, Gyoerkei A, Bogos B, Lazar V, Spohn R, Csoergoe B, Wagner A, Ruppin E, Pal C, and Papp B. 2014. Network-level architecture and the evolutionary potential of underground metabolism.pdf. Proc Natl Acad Sci U S A 111:11762-11767.

Ouwerkerk JP, de Vos WM, and Belzer C. 2013. Glycobiome: bacteria and mucus at the epithelial interface. Best Pract Res Clin Gastroenterol 27:25-38.

Pickard JM, Maurice CF, Kinnebrew MA, Abt MC, Schenten D, Golovkina TV, Bogatyrev SR, Ismagilov RF, Pamer EG, Turnbaugh PJ, and Chervonsky AV. 2014. Rapid fucosylation of intestinal epithelium sustains host-commensal symbiosis in sickness. Nature 514:638-641.

Quigley EM. 2013. Gut bacteria in health and disease. Gastroenterol Hepatol (N Y) 9:560-569.

Ramiro-Garcia, J, Hermes GDA, Giatsis C, Sipkema D, Zoetendal EG, Schaap PJ, and Smidt H. 2016. NG-Tax, a highly accurate and validated pipeline for analysis of 16S rRNA amplicons from complex biomes [version 1; referees: 2 approved with reservations, 1 not approved]. F1000Research 5:1791

Reichardt N, Duncan SH, Young P, Belenguer A, McWilliam Leitch C, Scott KP, Flint HJ, and Louis P. 2014. Phylogenetic distribution of three pathways for propionate production within the human gut microbiota. The ISME journal 8:1323-1335.

Ridlon JM, Kang DJ, and Hylemon PB. 2006. Bile salt biotransformations by human intestinal bacteria. J Lipid Res 47:241-259.

Robbe C, Capon C, Coddeville B, and Michalski JC. 2004. Structural diversity and specific distribution of O-glycans in normal human mucins along the intestinal tract. Biochem $J$ 384:307-316. 
759 Robbe C, Capon C, Maes E, Rousset M, Zweibaum A, Zanetta JP, and Michalski JC. 2003.

760

761

762

763

764

765

766

767

768

769

770

771

772

773

774

775

776

777

778

779

780

781

782

783

784

785

786

787

788

789

790

791

792

793

794

795

796

797

798

799

800

801

802

803

804

805

806

807

808

809

810

811

Evidence of regio-specific glycosylation in human intestinal mucins: presence of an acidic gradient along the intestinal tract. The Journal of biological chemistry 278:46337-46348.

Roggentin P, Gutschker-Gdaniec G, Schauer R, and Hobrecht R. 1985. Correlative properties for a differentiation of two Clostridium sordellii phenotypes and their distinction from Clostridium bifermentans. Zentralblatt fur Bakteriologie, Mikrobiologie, und Hygiene Series A, Medical microbiology, infectious diseases, virology, parasitology 260:319-328.

Rutherford JC. 2014. The emerging role of urease as a general microbial virulence factor. PLoS Pathog 10:e1004062.

Schwartz JJ, Gazumyan A, and Schwartz I. 1992. rRNA gene organization in the Lyme disease spirochete, Borrelia burgdorferi. Journal of bacteriology 174:3757-3765.

Scott KP, Gratz SW, Sheridan PO, Flint HJ, and Duncan SH. 2013. The influence of diet on the gut microbiota. Pharmacol Res 69:52-60.

Scott KP, Martin JC, Campbell G, Mayer CD, and Flint HJ. 2006. Whole-genome transcription profiling reveals genes up-regulated by growth on fucose in the human gut bacterium "Roseburia inulinivorans". Journal of bacteriology 188:4340-4349.

Sharma V, Prere MF, Canal I, Firth AE, Atkins JF, Baranov PV, and Fayet O. 2014. Analysis of tetra- and hepta-nucleotides motifs promoting -1 ribosomal frameshifting in Escherichia coli. Nucleic Acids Res 42:7210-7225.

Stahl M, Friis LM, Nothaft H, Liu X, Li J, Szymanski CM, and Stintzi A. 2011. L-fucose utilization provides Campylobacter jejuni with a competitive advantage. Proc Natl Acad Sci U S A 108:7194-7199.

Stams AJ, Van Dijk JB, Dijkema C, and Plugge CM. 1993. Growth of syntrophic propionateoxidizing bacteria with fumarate in the absence of methanogenic bacteria. Applied and environmental microbiology 59:1114-1119.

Suzek BE, Huang H, McGarvey P, Mazumder R, and Wu CH. 2007. UniRef: comprehensive and non-redundant UniProt reference clusters. Bioinformatics 23:1282-1288.

ter Braak CFJ, and Smilauer P. 2012. Canoco Reference Manual and User's Guide: Software for Ordination, Version 5.0. Ithaca, NY, USA: Microcomputer Power.

Tatusov RL, Koonin EV, Lipman DJ. 1997. A genomic perspective on protein families. Science 278:631-637.

Trapnell C, Hendrickson DG, Sauvageau M, Goff L, Rinn JL, and Pachter L. 2013. Differential analysis of gene regulation at transcript resolution with RNA-seq. Nat Biotechnol 31:4653.

UniProt-Consortium. 2014. Activities at the universal protein resource (UniProt). Nucleic Acids Res 42:D191-D198.

Van den Bogert B, Boekhorst J, Herrmann R, Smid EJ, Zoetendal EG, and Kleerebezem M. 2013a. Comparative genomics analysis of Streptococcus isolates from the human small intestine reveals their adaptation to a highly dynamic ecosystem. PloS one 8:e83418.

van den Bogert B, Erkus O, Boekhorst J, de Goffau M, Smid EJ, Zoetendal EG, and Kleerebezem M. 2013b. Diversity of human small intestinal Streptococcus and Veillonella populations. FEMS Microbiol Ecol 85:376-388.

van Hijum SA, Kralj S, Ozimek LK, Dijkhuizen L, and van Geel-Schutten IG. 2006. Structurefunction relationships of glucansucrase and fructansucrase enzymes from lactic acid bacteria. Microbiol Mol Biol Rev 70:157-176.

Vimr ER. 2013. Unified theory of bacterial sialometabolism: how and why bacteria metabolize host sialic acids. ISRN Microbiol 2013:816713.

Vimr ER, Kalivoda KA, Deszo EL, and Steenbergen SM. 2004. Diversity of microbial sialic acid metabolism. Microbiol Mol Biol Rev 68:132-153.

$\mathrm{Xu}$ J, Bjursell MK, Himrod J, Deng S, Carmichael LK, Chiang HC, Hooper LV, and Gordon JI. 2003. A genomic view of the human-Bacteroides thetaiotaomicron symbiosis. Science 299:2074-2076.

Yano K, Wada T, Suzuki, S Tagami K, Matsumoto T, Shiwa Y, Ishige T, Kawaguchi Y, 
812 Masuda K, Akanuma G, Nanamiya H, Niki H, Yoshikawa H, Kawamura F. 2013. Multiple rRNA operons are essential for efficient cell growth and sporulation as well as outgrowth in Bacillus subtilis. Microbiology; 159 (Pt 11):2225-2236.

Zhang Z, Geng J, Tang X, Fan H, Xu J, Wen X, Ma ZS, and Shi P. 2014. Spatial heterogeneity and co-occurrence patterns of human mucosal-associated intestinal microbiota. The ISME journal 8:881-893.

Zoetendal EG, Raes J, van den Bogert B, Arumugam M, Booijink CC, Troost FJ, Bork P, Wels M, de Vos WM, and Kleerebezem M. 2012. The human small intestinal microbiota is driven by rapid uptake and conversion of simple carbohydrates. The ISME journal 6:1415-1426. 


\section{Figure 1 (on next page)}

Circular map of the $R$. ilealis $\mathrm{CRIB}^{\top}$ genome

Both chromosome and non-mobilizable plasmid are shown. For the chromosome tracks from inside to outside are as follows: $1, \mathrm{GC}$ skew; $2, \mathrm{G}+\mathrm{C}$ content; 3 , RNAs [rRNAs (blue), tRNAs (orange) and ncRNAs (purple)]; 4, all predicted protein CDS [with predicted function (lightblue), hypothetical proteins and proteins to which only a domain of unknown function could be assigned (grey)]; 5, genes or gene clusters of interest [(mobile genetic elements (red), Cas proteins (pink), urease gene cluster (yellow), choloylglycine hydrolase (black), gene clusters involved in carbohydrate utilization (green)]. For the plasmid tracks from inside to outside are as follows: 1, GC skew; 2, G+C content; 3, all predicted CDS. 


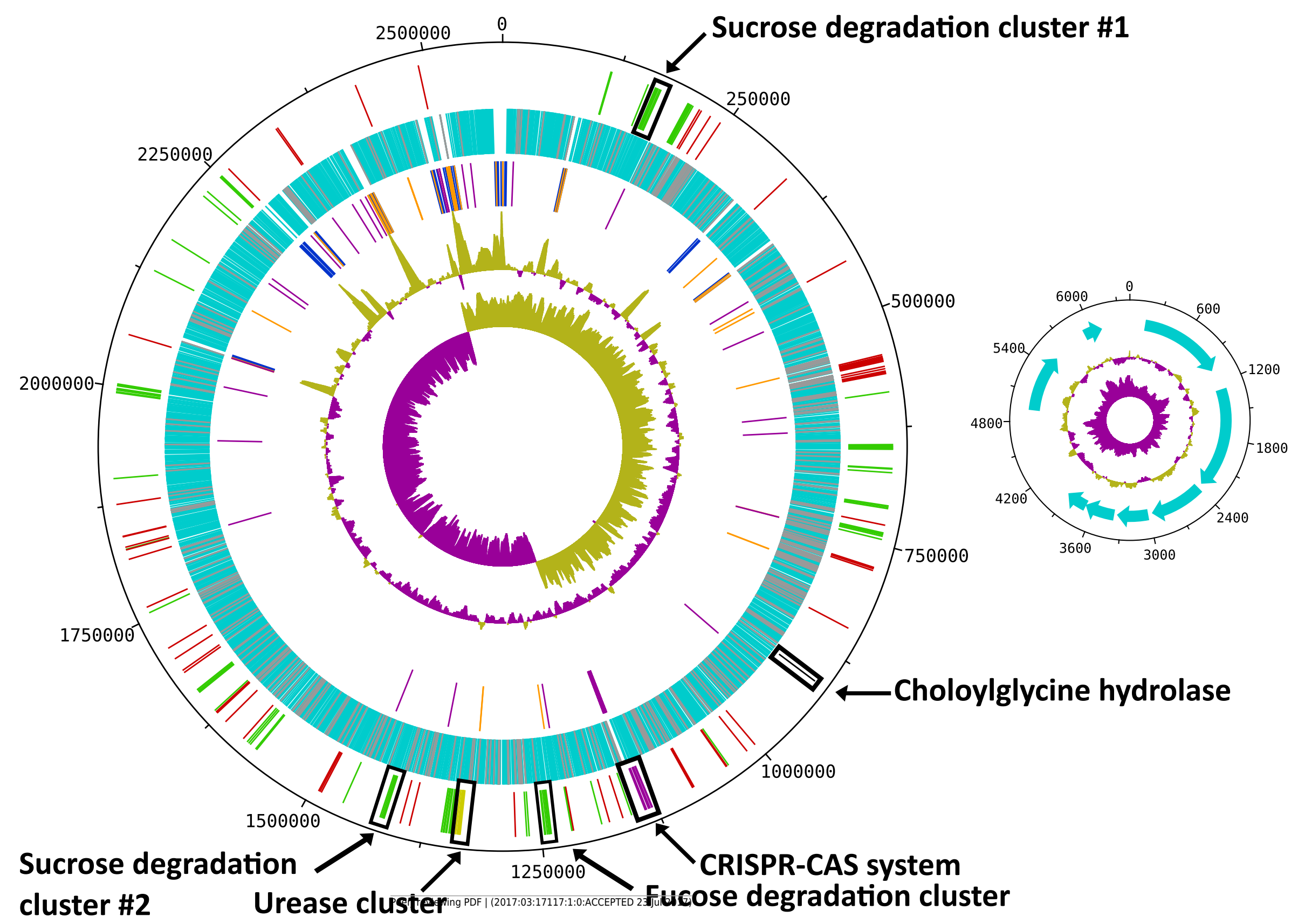




\section{Figure 2}

Principal component analysis of the transcriptomes of $R$. ilealis $\mathrm{CRIB}^{\top}$ grown on different carbohydrates (glucose, FOS and L-fucose) or in the absence of an additional carbon source (control).

First and second ordination axes are plotted, explaining $42.8 \%$ and $19.0 \%$ of the variability in the data set, respectively. Individual transcriptomes are symbol-coded by experimental condition: glucose (circles), FOS (squares), L-fucose (diamonds) and control (rectangles). The experimental conditions were used as supplementary variables as well and could explain $62.9 \%$ of the variation. 


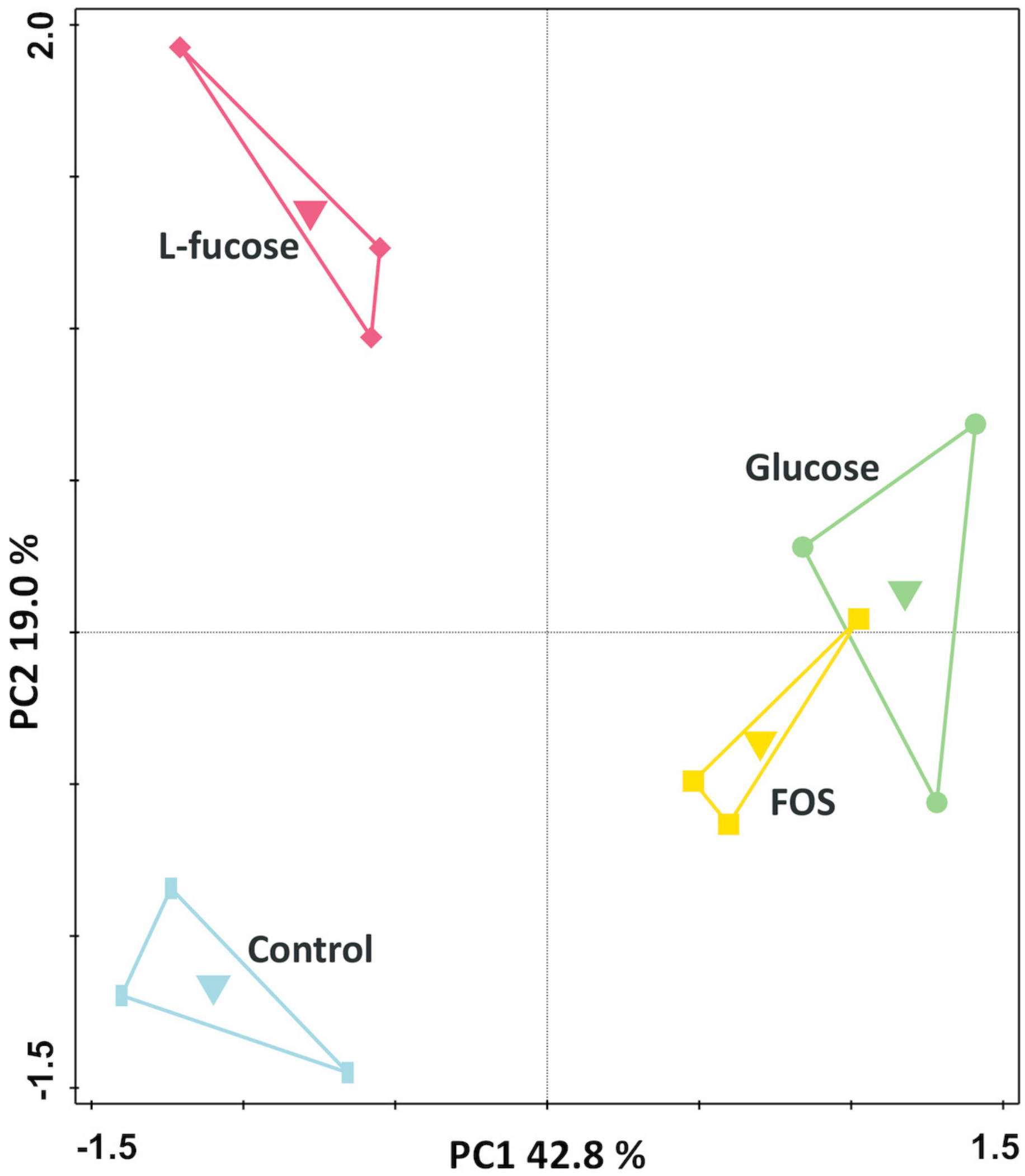


Figure 3

Heatmap of genes differentially expressed in at least one of the four conditions ( $\geq 1.5$ $\log 2$ (fold change) and q value $\leq 0.05$ ).

Colour coding by ratio to row mean. Key gene clusters are indicated. 

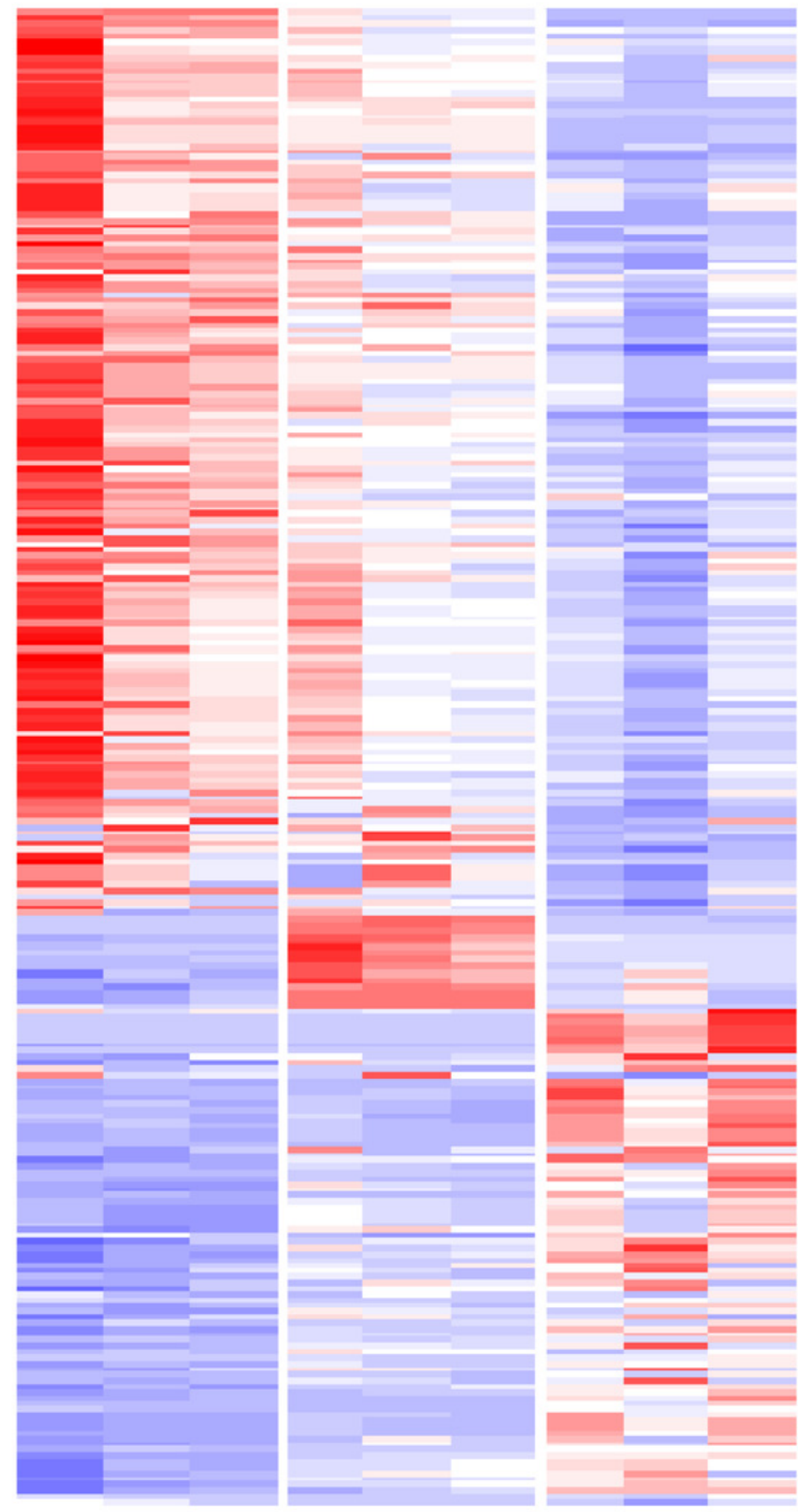

Glucose

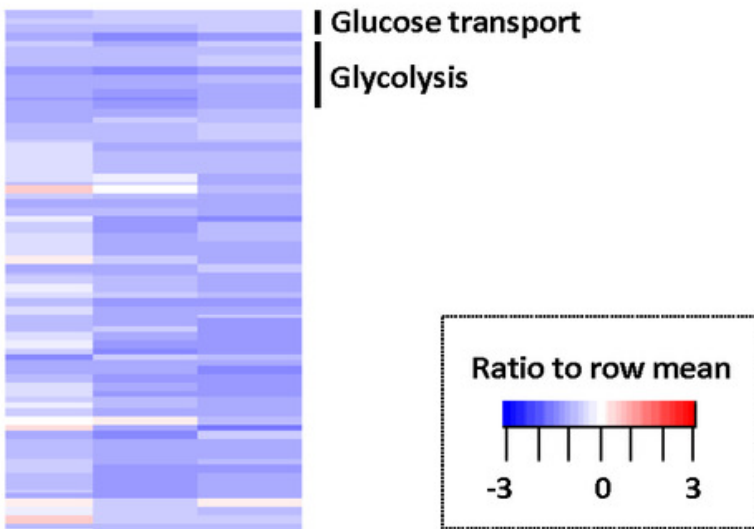

Glucose transport

Glycolysis

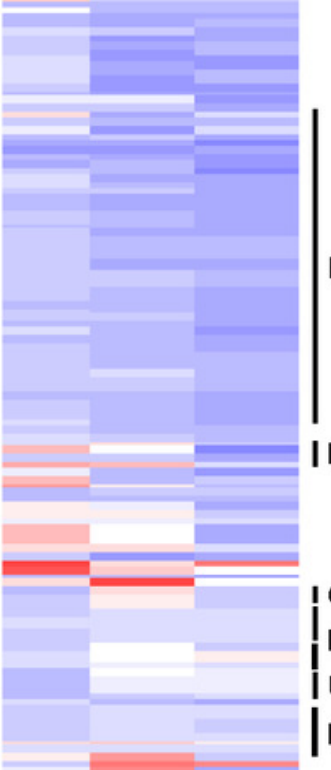

Ribosomal proteins

I Iron transport gene cluster

Cluster with alternative sigma factor FOS/sucrose degradation clusters I Unknown sugar transport cluster | L-fucose degradation cluster Sialic acid utilization cluster Cas proteins Glucuronide degradation cluster 1

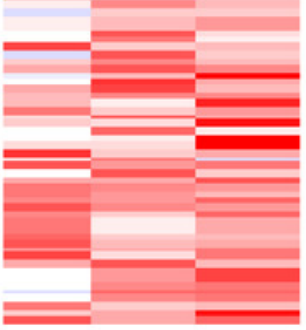

| Glucuronide degradation cluster 2 | Urease cluster

\section{Control}




\section{Figure 4(on next page)}

Schematic overview of the pathways involved in degradation of glucose, FOS and Lfucose in $R$. ilealis CRIB ${ }^{\top}$

$1^{A}$; PTS system glucose-specific EllA component (CRIB_2018); 1 ${ }^{B C}$, PTS system glucosespecific ElIBC component (CRIB_2017); 2 ${ }^{\mathrm{BC}}$, PTS system sucrose-specific ElIBC component (CRIB_1461); 3, B-fructofuranosidase with RDD family protein (CRIB_1459 and CRIB_1460); 4, fructokinase (CRIB_152 and CRIB_1458); 5; ABC-type transporter (CRIB_148-CRIB_150); 6, Bfructofuranosidase (CRIB_151); 7, glucokinase (CRIB_1849); 8, glucose 6-phosphate isomerase (CRIB_140); 9, fructose 1,6-bisphosphatase (CRIB_45 and CRIB_2020); 10, 6phosphofructokinase ; (CRIB_104); 11, fructose-bisphosphate aldolase (CRIB_2223); 12, triosephosphate isomerase (CRIB_189); 13, glyceraldehyde-3-phosphate dehydrogenase (CRIB_187); 14, phosphoglycerate kinase; 15, phosphoglycerate mutase (CRIB_1223) and 2,3-bisphosphoglycerate-independent phosphoglycerate mutase (CRIB_190); 16, enolase (CRIB_191); 17, pyruvate kinase (CRIB_105); 18, L-lactate dehydrogenase (CRIB_684); 19, formate acetyltransferase (CRIB_2141); 20, pyruvate-flavodoxin oxidoreductase (CRIB_2021); 21, phosphate acetyltransferase (CRIB_2171); 22, acetate kinase (CRIB_1927); 23, bifunctional aldehyde-alcohol dehydrogenase (CRIB_2231); 24, fatty aldehyde dehydrogenase (CRIB_2231); 25, L-fucose permease (CRIB_1294); 26, L-fucose isomerase (CRIB_1298); 27, L-fuculokinase (CRIB_1297); 28, L-fuculose phosphate aldolase (CRIB_1297); 29, lactaldehyde reductase (CRIB_1300); ?, possible mechanisms of external fructose accumulation (external degradation, or export). 


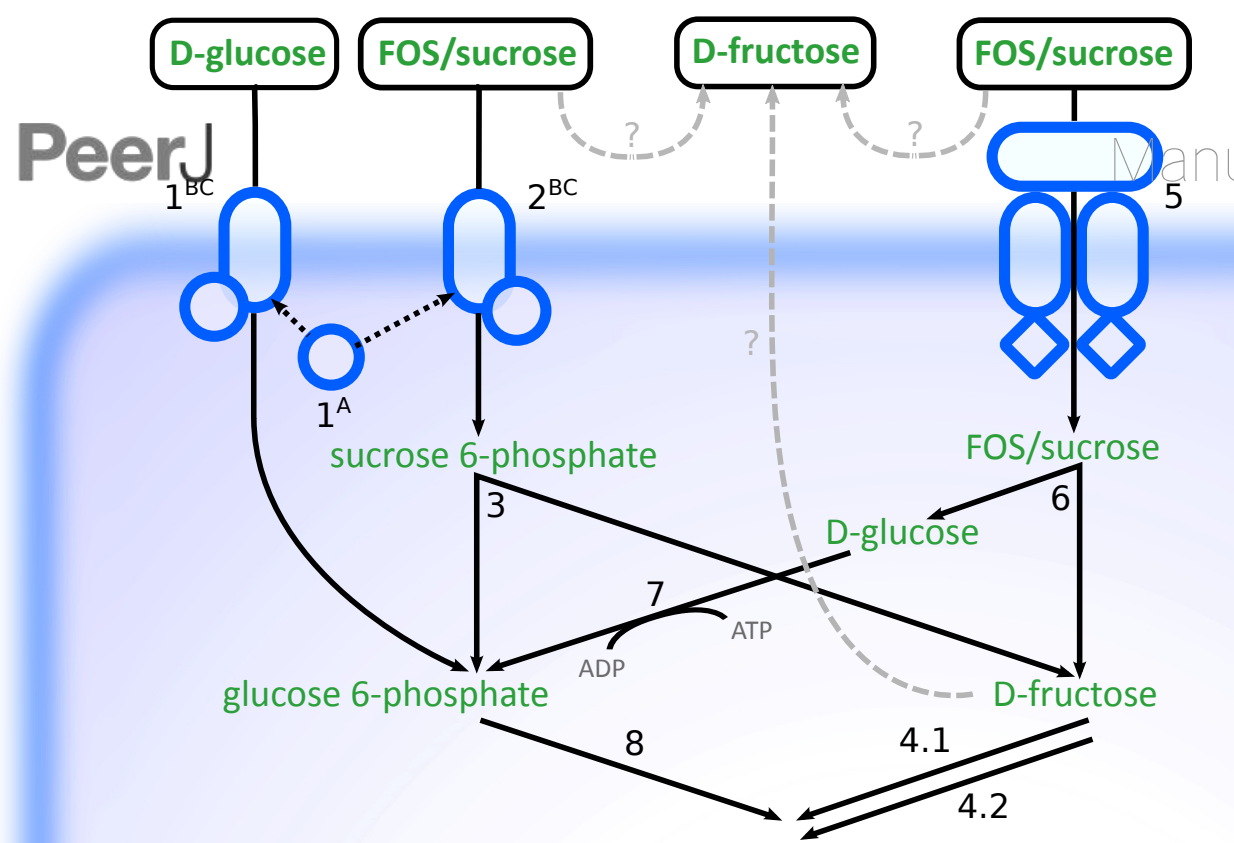

fructose 6-phosphate

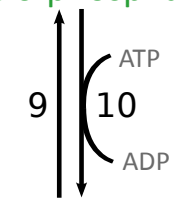

fructose 1,6-bisphosphate

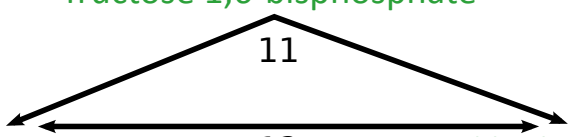

glyceraldehyde 3-phosphate

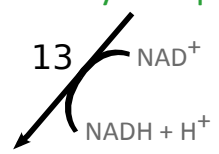

1,3-biphosphoglycerate<smiles>CC(C)C(C)(C)C</smiles>

3-phospho-D-glycerate

$$
15.2 \rrbracket 15.1
$$

2-phospho-D-glycerate

16
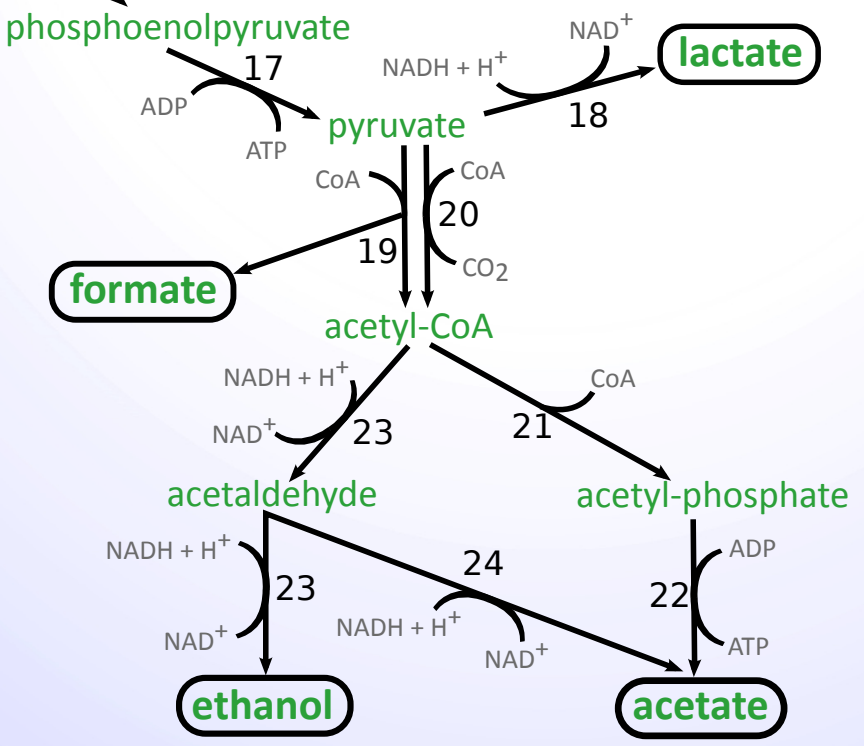

L-fucalose 1-phosphate

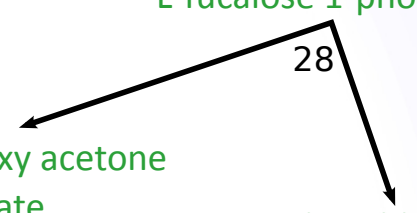

L-lactaldehyde

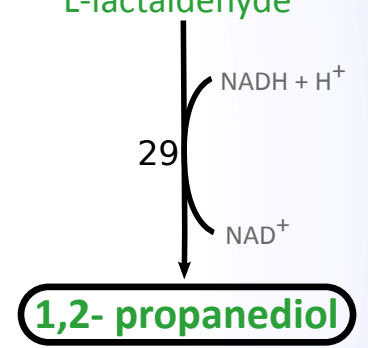


Table $\mathbf{1}$ (on next page)

General features of the $R$. ilealis $\mathrm{CRIB}^{\top}$ genome. 
1

\begin{tabular}{lll}
\hline & Chromosome & Plasmid \\
Size (bp) & $2,581,778$ & 6,145 \\
G+C content (\%) & 27.9 & 29.3 \\
Protein CDS & 2,351 & 8 \\
$\quad$ Pseudogenes & 12 & 0 \\
Coding density & 1.10 & 1.02 \\
Average gene size & 899 & 531 \\
(bp) & & \\
rRNA genes & & \\
$\quad$ 16S rRNA genes & 14 & 0 \\
$\quad$ 23S rRNA genes & $14 *$ & 0 \\
$\quad$ 5S rRNA genes & 14 & 0 \\
tRNAs & 109 & 0 \\
ncRNAs & 28 & 0 \\
CRISPR repeats & $1 * 71$ & 0 \\
\hline
\end{tabular}

* An additional 23S rRNA gene is expected in one of the gaps. 


\section{Table 2 (on next page)}

Fermentation end products of $R$. ilealis CRIB ${ }^{\top}$ produced during growth on different carbohydrates (glucose, FOS or L-fucose) or in basal medium in the absence of a carbon source (control condition)

Samples were obtained during mid-exponential phase ( 8-10 $\mathrm{h}$ incubation; used for transcriptome analysis) and in stationary phase ( $24 \mathrm{~h}$ incubation). For the control cultures, fermentation products are shown for the individual cultures separating the carbohydrates used for preconditioning of the inoculum. For the three other conditions, values represent means of triplicate cultures with standard deviations. N.D., not detected. 
1

\begin{tabular}{|c|c|c|c|c|c|c|c|c|c|c|}
\hline & \multicolumn{2}{|c|}{ Formate (mM) } & \multicolumn{2}{|c|}{ Acetate $(\mathrm{mM})$} & \multicolumn{2}{|c|}{ Propionate (mM) } & \multicolumn{2}{|c|}{ Lactate (mM) } & \multicolumn{2}{|c|}{ 1,2-propane-diol (mM) } \\
\hline & 8-10 h & $24 \mathrm{~h}$ & 8-10 h & $24 \mathrm{~h}$ & 8-10 h & $24 \mathrm{~h}$ & 8-10 h & $24 \mathrm{~h}$ & 8-10 h & $24 \mathrm{~h}$ \\
\hline $\begin{array}{l}\text { Control: basal medium } \\
\text { (glucose inoc.) } \\
\text { (FOS inoc.) } \\
\text { (L-fucose inoc) }\end{array}$ & $\begin{array}{l}3.2 \\
4.5 \\
4.8 \\
\end{array}$ & $\begin{array}{l}7.7 \\
9.2 \\
10.8 \\
\end{array}$ & $\begin{array}{l}2.0 \\
2.4 \\
2.3\end{array}$ & $\begin{array}{l}6.2 \\
7.4 \\
9.8 \\
\end{array}$ & $\begin{array}{l}2.0 \\
2.4 \\
2.3\end{array}$ & $\begin{array}{l}2.2 \\
2.9 \\
3.0\end{array}$ & $\begin{array}{l}\text { N.D. } \\
\text { N.D. } \\
\text { N.D. }\end{array}$ & $\begin{array}{l}\text { N.D. } \\
\text { N.D. } \\
\text { N.D. }\end{array}$ & $\begin{array}{l}\text { N.D. } \\
\text { N.D. } \\
1.0\end{array}$ & $\begin{array}{l}\text { N.D. } \\
\text { N.D. } \\
1.0\end{array}$ \\
\hline $\begin{array}{l}\text { Basal medium } \\
+ \text { glucose }(5 \% \mathrm{w} / \mathrm{v})\end{array}$ & $4.4 \pm 1.2$ & $28.2 \pm 4.3$ & $1.0 \pm 0.9$ & $16.3 \pm 2.2$ & $1.0 \pm 0.9$ & $1.3 \pm 0.1$ & N.D. & $3.0 \pm 0.7$ & N.D. & N.D. \\
\hline $\begin{array}{l}\text { Basal medium } \\
+ \text { FOS }(5 \% \mathrm{w} / \mathrm{v})\end{array}$ & $4.7 \pm 0.6$ & $27.3 \pm 2.5$ & $1.4 \pm 0.0$ & $17.7 \pm 1.4$ & $1.4 \pm 0.0$ & $1.6 \pm 0.1$ & N.D & $2.5 \pm 0.3$ & N.D. & N.D. \\
\hline $\begin{array}{l}\text { Basal medium } \\
+ \text { L-fucose }(5 \% \mathrm{w} / \mathrm{v}) \\
\end{array}$ & $6.7 \pm 0.1$ & $19.5 \pm 3.6$ & $2.8 \pm 0.1$ & $16.3 \pm 2.9$ & $2.8 \pm 0.1$ & $2.8 \pm 0.4$ & N.D. & N.D. & $1.3 \pm 0.1$ & $7.7 \pm 1.4$ \\
\hline
\end{tabular}

